\title{
Modelling and Verifying BDI Agents with Bigraphs
}

\author{
Blair Archibald ${ }^{\mathrm{a}}$, Muffy Calder $^{\mathrm{a}}$, Michele Sevegnani $^{\mathrm{a}}$, Mengwei Xu ${ }^{\mathrm{a}, *}$ \\ ${ }^{a}$ School of Computing Science, University of Glasgow, UK
}

\begin{abstract}
The Belief-Desire-Intention (BDI) architecture is a popular framework for rational agents; most verification approaches are based on reasoning about implementations of BDI programming languages. We investigate an alternative approach based on reasoning about BDI agent semantics, through a model of the execution of an agent program. We employ Milner's bigraphs as the modelling framework and present an encoding for the Conceptual Agent Notation (CAN) language - a superset of AgentSpeak featuring declarative goals, concurrency, and failure recovery.

We provide an encoding of the syntax and semantics of CAN agents, and give a rigorous proof that the encoding is faithful. Verification is based on the use of mainstream software tools including BigraphER, and a small case study verifying several properties of Unmanned Aerial Vehicles (UAVs) illustrates the framework in action. The executable framework is a foundational step that will enable more advanced reasoning such as plan preference, intention priorities and trade-offs, and interactions with an environment under uncertainty.
\end{abstract}

Keywords: BDI Agents, Modelling, Verification, Bigraphs

\section{Introduction}

The Belief-Desire-Intention (BDI) 1] architecture is a popular and wellstudied rational agent framework and forms the basis of, among others, AgentSpeak [2], Artificial Autonomous Agents Programming Language (3APL) [3], A Practical Agent Programming Language (2APL) [4], Jason [5], and Conceptual Agent Notation (CAN) [6]. In a BDI agent, the (B)eliefs represent what the agent knows, the $(D)$ esires what the agent wants to bring about, and the (I)ntentions those desires the agent has chosen to act upon. BDIs have been very successful in many areas such as business [7], healthcare [8], and engineering [9].

The deployment of autonomous systems in real-world applications raises concerns of trustworthiness and safety, for example in scenarios such as autonomous

\footnotetext{
${ }^{*}$ Corresponding Author

Email addresses: blair.archibald@glasgow.ac.uk (Blair Archibald), muffy.calder@glasgow.ac.uk (Muffy Calder), michele.sevegnani@glasgow.ac.uk (Michele Sevegnani), mengwei.xu@glasgow.ac.uk (Mengwei Xu)
} 


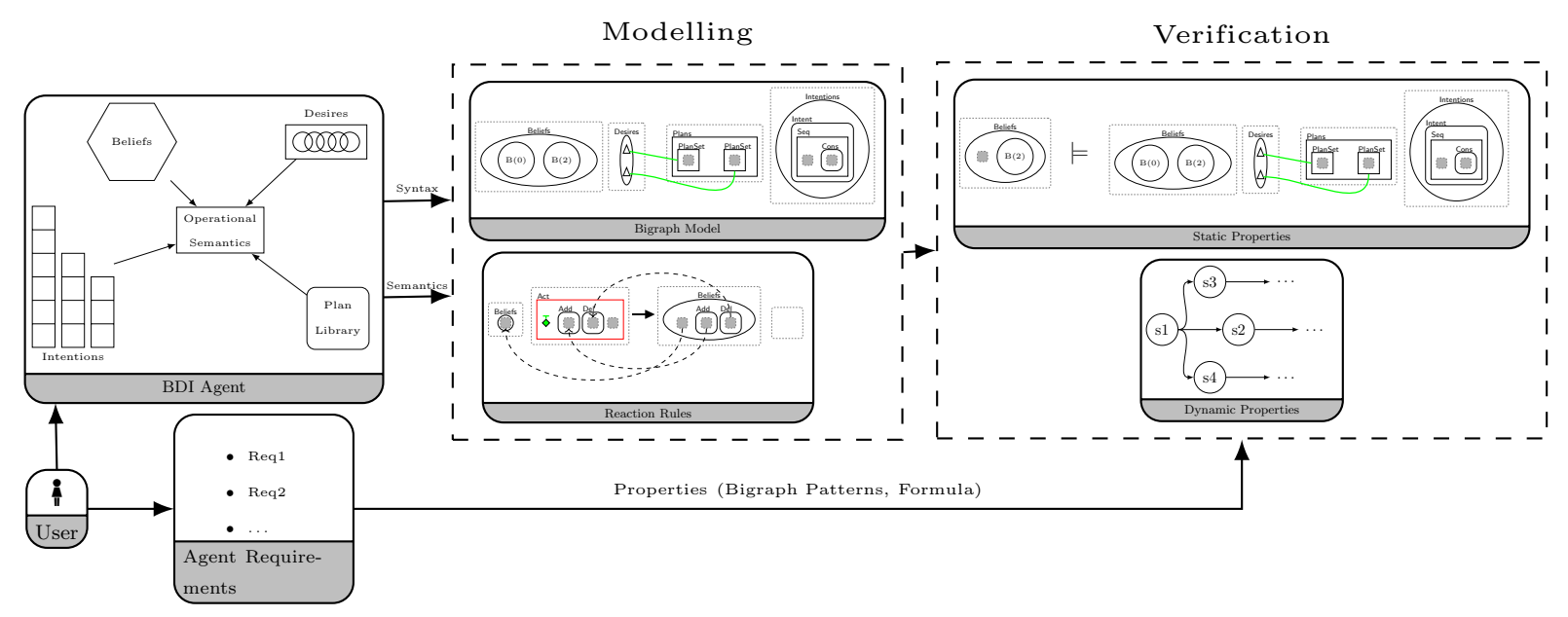

Figure 1: Modelling and Verification Framework for BDI Agents.

control in space [10] and human-robot interaction in healthcare [11]. There is a growing demand for verification techniques to aid analysis of behaviours in increasingly complex and critical domains, and there has been a proliferation of techniques and languages supporting BDI agent verification. Most of these approaches, however, focus on verifying the implementations of BDI programming languages. For example, recent work implements a BDI agent programming language as a set of Java classes - the Agent Infrastructure Layer (AIL) [12] - that can be verified using the Java PathFinder [13] program model checker. While verifying an implementation tells you how the system will operate, it might not correspond to how it should operate with respect to the semantics of the given BDI programming language.

We present an approach that reasons on the semantics of the BDI programming language (rather than the implementation of it) through a mathematical model of the execution of the agent program, i.e. verified executable semantics.

Our approach models and verifies BDI agents, specified in the CAN language, by encoding them as an instance of Milner's Bigraphical Reactive Systems (BRS) [14]. CAN features a high-level agent programming language that captures the essence of BDI concepts without describing implementation details such as data structures. As a superset of AgentSpeak, CAN includes advanced BDI agent behaviours such as reasoning with declarative goals, concurrency, and failure recovery. Importantly, although we focus on CAN, the language features are similar to those of other mainstream BDI languages and the same modelling techniques would apply to other BDI programming languages.

Bigraphs provide a meta-modelling framework that has been developed as a unifying theory for existing calculi, e.g. $\pi$-calculus [15]. As a graph-based rewriting formalism, over rules called reaction rules, Bigraphs provide an intuitive diagrammatic representation, which is ideal for visualising the execution 
process of CAN. Support for sharing, probabilistic, and conditional rewriting extensions [16, 17, 18] in Bigraphs further enables the increases of expressiveness (when required) for more advanced reasoning, e.g. probabilistic reasoning. For analysis, BigraphER [19] is a freely available tool for working with bigraph models including rewriting, verification based on bigraph patterns, and transition system export to model checking tools [20].

Our bigraph encoding of the CAN language includes: i) a structural encoding that maps the syntax of CAN (e.g. beliefs, plans, and intentions) into equivalent bigraphs, and ii) an encoding of the operational semantics of CAN as a set of reaction rules. We provide a correctness proof that the translation of CAN semantics into reaction rules is faithful.

The framework is depicted in Fig. 1. On the left we have the BDI agents and agent requirements - logical formulas. In the middle, Modelling, the agents are translated into bigraphs that capture the structural elements and reaction rules that capture their dynamics. Once we have encoded the agent into bigraphs, we use the model to perform verification (on the right) of user-specified agent requirements. Verification takes two forms: checking static properties of a state (the current bigraph representing an agent at some point in its execution) through bigraph patterns, and checking dynamic properties, expressed as temporal logic properties, against the transition system generated by BigraphER. Finally, the user can employ BigraphER simply to "run" their agent model with different initial settings.

We illustrate the framework with a small case study based on Unmanned Aerial Vehicles (UAVs).

We make the following contributions:

- an encoding of the CAN language and operational semantics in bigraphs, using regions to represent the perspectives of Belief, Desire, Intention, and Plan,

- proof that the encoding is faithful by showing each CAN semantic rule is encoded by a (finite) sequence of reaction rules,

- an illustration of our framework in a UAV case study,

- a reflection on aspects of CAN based on insights gained from the encoding,

- an overview of how we will build upon this foundation in future to reason about plan selection, intention tradeoffs and priorities, and interactions with an uncertain environment.

The paper is organised as follows: in Section 2, we recall preliminaries of BDI agents in the CAN language and bigraphs; in Section 3 , we provide the structural encoding that maps the syntax of CAN into equivalent bigraphs; in Section 4, we present a comprehensive review of the core semantics of CAN (excluding concurrency and declarative goals) and, in particular, how the operation of CAN semantics can be viewed as AND/OR trees. In Section 5 we 
encode the semantics given in Section 4 and in Section 6, we present the semantics for concurrency and declarative goals and provide their bigraphical encodings. In Section 7 and Section 8, we illustrate our framework with examples and in Section 9, we reflect on aspects of CAN. In Section 10 we discuss related work; in Section 11 we lay out the our plans of for future extensions to this work; we conclude in Section 12

\section{Preliminaries}

We give an overview of BDI agents, described in the Conceptual Agent Notation (CAN) language, as well as Bigraphs and Bigraphical Reactive Systems (BRS).

\subsection{BDI Agents}

A BDI agent has an explicit representation of beliefs, desires, and intentions. The beliefs correspond to what the agent believes about the environment, while the desires are a set of external events that the agent can respond to. To responds to those events, the agent selects an appropriate plan (given its beliefs) from the pre-defined plan library and commits to the selected plan by turning it into a new intention.

CAN is a superset of AgentSpeak 2] featuring the same core operational semantics, along with several additional appealing features: declarative goals, concurrency, and failure handling. In the following, we introduce the syntax of CAN, the semantics are given in Section 4

A CAN agent consists of a belief base $\mathcal{B}$ and a plan library $\Pi$. The belief base $\mathcal{B}$ is a set of formulas encoding the current beliefs. Without loss of generality, we specify our belief base following the logical language in AgentSpeak [2] that takes the form $\varphi::=b|\neg b|\left(\varphi_{1} \wedge \varphi_{2}\right) \mid$ true $\mid$ false. More complex logics are possible but are outwith the scope of this paper, i.e. we show how to encode general BDI agents in bigraphs, not how to encode specific logics. All that we assume for any chosen logical language is that it has belief operators to check whether a belief formula $\varphi$ follows from the belief base (i.e. $\mathcal{B} \models \varphi$ ), to add a belief atom $b$ to a belief base $\mathcal{B}$ (i.e. $\mathcal{B} \cup\{b\}$ ), and to delete a belief atom from a belief base (i.e. $\mathcal{B} \backslash\{b\}$ ).

A plan library $\Pi$ contains the operational procedures of an agent and is a finite collection of plans of the form $P l=e: \varphi \leftarrow P$ with $P l$ the plan identifier, $e$ the triggering event, $\varphi$ the context condition, and $P$ the plan-body. The triggering event $e$ specifies why the plan is triggered, the context condition $\varphi$ determines when the plan-body $P$ is able to handle the event. We denote the triggering event of a plan $P l$ trigger $(P l)$ and we call $E=\{\operatorname{trigger}(P l) \mid P l \in \Pi\}$ the event set that the agent knows how to respond to (i.e. it has plans for response - though it might be the case none are applicable). For convenience, we call the set of events from the external environment the external event set, denoted $E^{e}$. Finally, the remaining events (which occur as a part of the planbody) are either sub-events or internal events. 
By convention (e.g. in [5]), the plan-body $P$ in a plan $P l=e: \varphi \leftarrow P$ may be referred to as the program or agent program and has the following syntax:

$$
P::=a c t|? \varphi|+b|-b| e\left|P_{1} ; P_{2}\right| P_{1} \| P_{2} \mid \operatorname{goal}\left(\varphi_{s}, P, \varphi_{f}\right)
$$

with act an action, ? $\varphi$ a test for $\varphi$ entailment in the belief base, $+b$ and $-b$ represent belief addition and deletion, and $e$ is a sub-event (i.e. internal event). Actions act take the form act $=\varphi \leftarrow\left\langle\phi^{+}, \phi^{-}\right\rangle$, where $\varphi$ is the pre-condition, and

$\phi^{+}$and $\phi^{-}$are the addition and deletion sets (resp.) of belief atoms, i.e. a belief base $\mathcal{B}$ is revised with addition and deletion sets $\phi^{+}$and $\phi^{-}$to be $\left(\mathcal{B} \backslash \phi^{-}\right) \cup \phi^{+}$. In addition, there are composite programs $P_{1} ; P_{2}$ for sequence and $P_{1} \| P_{2}$ for interleaved concurrency. Finally, a declarative goal program $\operatorname{goal}\left(\varphi_{s}, P, \varphi_{f}\right)$ expresses that the declarative goal $\varphi_{s}$ should be achieved through program $P$, failing if $\varphi_{f}$ becomes true, and retrying as long as neither $\varphi_{s}$ nor $\varphi_{f}$ is true (see in [21] for details). Additionally, there are auxiliary program forms that are used internally when assigning semantics to programs, namely nil, the empty program, and $P_{1} \triangleright P_{2}$ that executes $P_{2}$ if the case that $P_{1}$ fails.

When a plan $P l=e: \varphi \leftarrow P$ is selected to respond to an event, its planbody $P$ is adopted as an intention in the intention base $\Gamma$ (a.k.a. the partially executed plan-body). Finally, we assume a plan library does not have recursive plans (thus avoiding potential infinite state space).

\subsubsection{Running Example - Conference Travel Agent}

For illustration, we give a classic example - arranging a conference trip - as shown in Fig. 2.

A BDI agent desires to arrange a conference trip, denoted by an external event $e_{1}$. We assume there are only two ways to travel to the conference. The first way is to travel by car, given by the plan $P l_{1}=e_{1}: \varphi_{1} \leftarrow$ act $_{1}$; act 2 s.t. $\varphi_{1}=b_{1} \wedge b_{2}$. The plan $P l_{1}$ expresses that if the agent believes it owns a car (i.e. $b_{1}$ ) and the venue is in the driving distance (i.e. $b_{2}$ ), it can start the car and drive all the way to the venue. To specify the actions, we have $a c t_{1}=b_{3} \leftarrow\left\langle\left\{b_{4}\right\}, \emptyset\right\rangle$ and $a c t_{2}=b_{4} \leftarrow\left\langle\left\{b_{5}\right\}, \emptyset\right\rangle$. For example, the action act $_{1}$ expresses that if the car is functional (i.e. $b_{3}$ ) and after executing $a c t_{1}$, the belief of the engine being on (i.e. $b_{4}$ ) will be added while deleting nothing from the belief base.

The second way is to travel by air, given by the plan $P l_{2}=e_{1}: \varphi_{2} \leftarrow$ $a^{a c t_{3}} ; e_{2} ; a c t_{4}$ s.t. $\varphi_{2}=b_{6} \wedge b_{7}$. This plan expresses that if the budget allows (i.e. $b_{6}$ ) and there is a flight (i.e. $b_{7}$ ), the agent can book the ticket first, then post internally a sub-event to actually travelling by plane, and go to the venue after landing. For actions, we have act $_{3}=$ true $\leftarrow\left\langle\left\{b_{8}\right\}, \emptyset\right\rangle$ and act $_{4}=b_{9} \leftarrow\left\langle\left\{b_{5}\right\}, \emptyset\right\rangle$. To address the sub-event $e_{2}$, we have plan $P l_{3}=e_{2}: \varphi_{3} \leftarrow$ act $_{5} ;$ act $_{6}$ s.t. $\varphi_{3}=b_{8} . P l_{3}$ expresses that if the agent believes the flight has been booked, it can go to the airport and fly by plane. Also, we have act $_{5}=b_{8} \leftarrow\left\langle\left\{b_{10}\right\}, \emptyset\right\rangle$ and $a c t_{6}=b_{10} \leftarrow\left\langle\left\{b_{9}\right\},\left\{b_{8}, b_{10}\right\}\right\rangle$. In particular, action $a c t_{6}$ indicates that if at airport (i.e. $b_{10}$ for at_airport), after the flight it will add the belief atom $b_{9}$ for flight_landed, and delete both belief atoms $b_{8}$ for flight_booked and $b_{10}$ for at_airport. 


\section{Conference Travel Agent}

1 Belief base: $b_{1}, b_{2}, b_{6}, b_{7}$

2 External events: $e_{1}$

3 Plan library:

$4 P l_{1}=e_{1}: \varphi_{1} \leftarrow a c t_{1} ; a c t_{2}$ s.t. $\varphi_{1}=b_{1} \wedge b_{2}$

$5 \mathrm{Pl}_{2}=e_{1}: \varphi_{2} \leftarrow a c t_{3} ; e_{2} ;$ act $_{4}$ s.t. $\varphi_{2}=b_{6} \wedge b_{7}$

$6 \mathrm{Pl}_{3}=e_{2}: \varphi_{3} \leftarrow \operatorname{act}_{5} ; a c t_{6}$ s.t. $\varphi_{3}=b_{8}$

7 Actions

8 act $_{1}=b_{3} \leftarrow\left\langle\left\{b_{4}\right\}, \emptyset\right\rangle$

9 act $_{2}=b_{4} \leftarrow\left\langle\left\{b_{5}\right\}, \emptyset\right\rangle$

10 act $_{3}=$ true $\leftarrow\left\langle\left\{b_{8}\right\}, \emptyset\right\rangle$

$11 a^{a c t_{4}}=b_{9} \leftarrow\left\langle\left\{b_{5}\right\}, \emptyset\right\rangle$

12 act $_{5}=b_{8} \leftarrow\left\langle\left\{b_{10}\right\}, \emptyset\right\rangle$

13 act $_{6}=b_{10} \leftarrow\left\langle\left\{b_{9}\right\},\left\{b_{8}, b_{10}\right\}\right\rangle$

where $e_{1}$ stands for conference_travelling, $e_{2}$ for get_onboard,

$a c t_{1}$ for start_car, act ${ }_{2}$ for driving, act ${ }_{3}$ for book_flight,

$a c t_{4}$ for go_to_venue, $a c t_{5}$ for go_to_airport, $a c t_{6}$ for flying,

$b_{1}$ for own_car, $b_{2}$ for driving_distance, $b_{3}$ for car_functional,

$b_{4}$ for engine_on, $b_{5}$ for at_venue, $b_{6}$ for budget_allowed,

$b_{7}$ for flight_available, $b_{8}$ for flight_booked, $b_{9}$ for flight_landed,

and $b_{10}$ for at_airport.

Figure 2: A BDI agent for conference travelling 
Table 1: Bigraph components and operations.

$\begin{array}{lcl}\text { Component/Operation } & \text { Algebraic Form } & \text { Diagrammatic Form } \\ \text { Entity of arity } 1 & \mathrm{~K}_{a} \\ \text { Name closure } & / a \mathrm{~K}_{a} \\ \text { Site } & \text { id } \\ \text { Region } & \text { Act.B.id } \\ \text { Nesting } & \mathrm{C}_{x} \text {.id } \| \mathrm{D}_{x} . \text { id } \\ \text { Parallel product } & \mathrm{C}_{x} \text {.id } \mid \mathrm{D}_{x} \text {.id }\end{array}$

We define the initial belief base to be $\mathcal{B}=\left\{b_{1}, b_{2}, b_{6}, b_{7}\right\}$. This expresses the agent believes that it owns a car $\left(b_{1}\right)$, the venue is in the driving distance $\left(b_{2}\right)$, the budget is sufficient for flight $\left(b_{6}\right)$, and there is a flight available $\left(b_{7}\right)$.

\subsection{Bigraphs}

Bigraphs are a universal modelling language, introduced by Milner 22], for both modelling ubiquitous systems and as a unifying theory for many existing calculi for concurrency and mobility. A bigraph consists of a pair of relations over the same set of entities: a directed forest representing topological space in terms of containment, and a hyper-graph expressing the interactions and (non-spatial) relationships among entities. Each entity is assigned a type, which determines its arity (i.e. number of links), and whether it is atomic (i.e. it cannot contain other entities). For the purpose of presenting our approach, we provide only an informal overview of bigraphs. A concise semantics can be found elsewhere e.g. [22].

Bigraphs can be described in algebraic terms or with an equivalent diagrammatical representation as shown in Table 1. In general, bigraphs permit any kind of shape (sometimes coloured) for typed entities. We allow entities to be parameterised, i.e. $\mathrm{K}(n)$, allowing them to represent families of entities. Entities can be connected through green links. Names 1 allow links (or potential links) to bigraphs in an external environment or context, and are written above the bigraph. Unconnected links are closed and drawn as a closed-off link. Grey

\footnotetext{
${ }^{1}$ Specifically outer-names.
} 


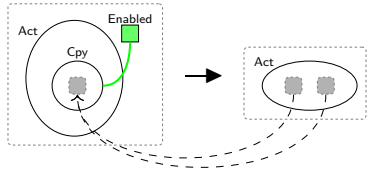

(a) Reaction rule copy.

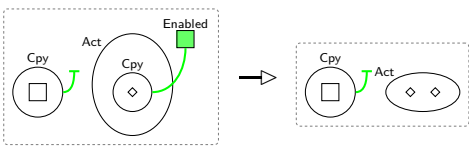

(b) Result of applying reaction rule copy.

Figure 3: Example bigraph and reaction rule.

rectangles are called sites that indicate parts of the model that have been abstracted away. In other words, an entity containing a site can contain zero or more entities of any kind. Finally, a dashed rectangle denotes a region of adjacent parts of the system.

Topological placement of entities is described using: nesting that defines the containment relation on entities; merge product that places two entities side-byside at the same hierarchical level; and parallel product that places entities in separate regions (allowing them to be at different levels of the hierarchy). In both merge and parallel product, bigraphs are linked on common names. An overview of the bigraph components and operations are given in Table 1.

Example bigraphs are shown in Fig. 3 where the left and right hand side of arrows represent different bigraphs. Written algebraically the left-side of Fig. 3b is:

$$
\left(/ l \mathrm{Cpy}_{l} . \text { Square.1) |/c (Act.Cpy } . \text { Diamond.1 | Enabled } .1\right)
$$

\subsection{Bigraphical Reactive Systems}

A bigraph represents a system at a single point in time. To allow models to evolve over time we can specify a Bigraphical Reactive System (BRS) that acts as a rewriting system. A BRS consists of a set of reaction rules of the form $L \rightarrow R$, where $L$ and $R$ are bigraphs. Intuitively, a bigraph $B$ evolves to $B^{\prime}$ by matching and rewriting an occurrence of $L$ in $B$ with $R$. Such a reaction is indicated with $B \rightarrow B^{\prime}$. We use $\rightarrow+$ to denote one or more applications of a rule, and $\longrightarrow$ to denote zero or more rule applications. We also write $\stackrel{\text { rule }}{\longrightarrow}$ to identify the reaction rule being applied to generate the transition. If no name is specified we assume any rule applied. Reaction rules can be parameterised when they are defined over entities with parameterised types, i.e. a rule $\mathbf{r}(k)$ for all values of $k$. The transition system of a BRS is a (possibly infinite) graph whose vertices are bigraphs representing the reachable states and whose edges represent reactions over bigraphs.

An example reaction rule, copy, is shown in Fig. 3a. This models a reaction which should copy elements of a site found within an entity Cpy only if that Cpy is inside an Act and connected through a link to an Enable entity. The use of a site abstracts from the specific entities to be copied. To allow copying (and deletion), reaction rules can be augmented with instantiation maps that determine a mapping between sites on the left and right-hand side of a reaction rule. Instantiation maps are denoted graphically as dashed arrows mapping 
sites in the right-hand side $R$ to sites in left-hand side $L$. The instantiation map is omitted from a rule definition when it is an identity. For example, the outcome of applying the reaction rule copy to a larger starting bigraph is shown in Fig. 3b. Notice that as the copy on-the-left is not within an Act, there is only one match.

We also use conditional bigraphs [18] that allow application conditions to specify contextual requirements within the rewrite system. For example, we can exclude certain bigraphs appearing within sites of the left-hand-side of a rule. We write conditions in the form: if $\langle-, \quad, \downarrow\rangle$ where the - indicates a negative conditions, the black circle represents an arbitrary bigraph we want to disallow, and $\downarrow$ indicates we should disallow from the site 2 . Importantly the bigraph in the condition cannot appear anywhere in the site, including nested below other entities. When more than one condition is specified for a reaction (separated by commas) they must all hold for the rule to apply.

Furthermore, rule priorities can be introduced by defining a partial ordering on the reaction rules of a BRS, as implemented in 23]. A reaction rule of lower priority can be applied only if no rule of higher priority is applicable. We write $r_{1}<r_{2}$ when $r_{2}$ has higher priority than $r_{1}$. This notation extends to sets in the natural manner, e.g. $\left\{r_{1}, r_{3}\right\}<\left\{r_{2}, r_{4}\right\}$, where rules in the same set have the same priority.

A common approach for verifying a BRS is through (bounded) model checking on its transition system. To allow labelling of states, which are themselves bigraphs, we define predicates as bigraph patterns. Informally, a pattern can be seen as a left-hand-side of a reaction rule, i.e. the input to the matching problem. A single state may have multiple labels if multiple patterns occur in it. Patterns can also be combined with standard Boolean operators to form logical formulae.

\section{Encoding BDI Agents in Bigraphs}

We define the structural encoding that maps the syntax (e.g. plans and actions) of a CAN BDI agent into equivalent bigraphs.

Recall a BDI agent is specified by a belief base $\mathcal{B}$ consisting of a set of belief atoms, e.g. $\mathcal{B}=\left\{b_{1}, \ldots, b_{n}\right\}$, a set of events (i.e. desires) the agent responds to, and a plan library $\Pi$ containing plans in form of $P l=e: \varphi \leftarrow P$. As the agent executes, plan-bodies selected for addressing desires become the intentions of the agent.

We take a multi-perspective approach (as introduced in [20]) in which perspectives are represented by separate and parallel regions. Mirroring the core components of a BDI agent, we employ four perspectives: Belief that handles knowledge storage and updates; Desire that manages the external events; Intention that captures the current execution states of plan-bodies; and Plan that

\footnotetext{
${ }^{2}$ Conditional bigraphs also allows positive, and contextual conditions, however we do not use these here.
} 
holds instructions for the agent on how to bring about its desires (i.e. how to respond to specific events). This approach allows us to separate design concerns, to be explicit how and when concerns interact, and to visualise them naturally, as shown in Fig. 1. It also facilitates model extension, for example we could in future add perspectives for the external (uncertain) environment, or we could replace the Beliefs perspective with one that allows more complex logic formulas.

The entities in the bigraph model for the syntax of a BDI agent are given in Table 2, grouped by the four perspectives. For each entity we give the algebraic form as well as structural information in the form of valid parents and linked entities. The only atomic entities, i.e. that cannot nest other entities, are belief atoms $\mathrm{B}(n)$, logical constant e.g. false, and events $\mathrm{E}_{e}$. Detailed information on the role for each of these entities is given as we introduce the encoding.

We define an encoding $\llbracket \cdot \rrbracket: B D I \rightarrow \mathbf{B g}(\mathcal{K})$ that maps the syntax of a BDI agent - including beliefs, desires, intentions, and plans - to an equivalent bigraph, where $\mathcal{K}$ denotes the set of all entity types in Table 2 , No information is lost through $\llbracket \cdot \rrbracket$ and it is possible to define the inverse encoding $\llbracket \cdot \rrbracket^{-1}$ establishing an equivalence. Although the inverse is easy to define, some cases are context dependent, e.g. rules 13 and 14 related to belief atoms in Fig. 4 have the same bigraph representation but always appear in distinct contexts (pre-conditions and action outcomes respectively 3 . For brevity we omit the details of the inverse encoding.

The encoding is defined inductively as shown in Fig. 4. To aid explanation, we give the encoding in two parts. In Fig. 4a, the encoding of agent belief, desire and intention structures are given. In the second part, the encoding of plans, in particular the plan-bodies, of an agent are provided in Fig. 4b. The parts are not distinct e.g. the plans within the plan library are encoded using the encoding of plan-bodies. We use $\prod \mathrm{M} \stackrel{\text { def }}{=} \mathrm{M}|\ldots| \mathrm{M}$ to denote iterated merge product. In the next few sections, we explain the (numbered) rules in Fig. 4.

\subsection{Encoding of Beliefs, Desires, and Intentions}

Rule 1 ensures the top-level components of an agent - beliefs, desires, intentions, and plan library - are mapped to separate perspective (region) in the bigraph.

We assume all belief formulas $\varphi$ are expressed in propositional logic. Recall that the convention in AgentSpeak for the belief base is $\varphi::=b|\neg b|\left(\varphi_{1} \wedge \varphi_{2}\right) \mid$ true $\mid$ false. For convenience, the parameterised entities $\mathrm{B}(i)$ (rule 3 ) are used for both positive and negative atoms: $b$ or $\neg b$ (e.g. $\llbracket b \rrbracket=\llbracket b_{0} \rrbracket=\mathrm{B}(0), \llbracket \neg b \rrbracket=$ $\left.\llbracket b_{1} \rrbracket=\mathrm{B}(1)\right)$. Using this, all formulas can be constructed in pure conjunctive form, i.e. $\varphi=b_{1} \wedge \cdots \wedge b_{n}$. We allow logical constants for true and false representing formulas that are always/never entailed, e.g. an action with precondition false never executes. In the bigraph model, we only assign an entity

\footnotetext{
${ }^{3}$ The inverse of true is a special case as we may map either to the truth term or an empty context set. However both options give rise to behaviourally equivalent agents.
} 
Table 2: Bigraph entities for BDI syntax encoding

\begin{tabular}{|c|c|c|c|c|}
\hline Description & Entity & Parent(s) & LinksTo & $\begin{array}{c}\begin{array}{c}\text { Diagrammatic } \\
\text { Form }\end{array} \\
\end{array}$ \\
\hline Belief Base & Beliefs & & & $\overbrace{}^{\text {Beliefs }}$ \\
\hline Belief Atoms & $\mathrm{B}(n)$ & $\{$ Beliefs, Pre, Add, Del $\}$ & & \\
\hline Logical False & false & $\{$ Beliefs, Pre $\}$ & & false \\
\hline $\begin{array}{l}\text { Desire Set } \\
\text { Event } \\
\end{array}$ & $\begin{array}{c}\text { Desires } \\
\mathrm{E}_{e} \\
\end{array}$ & $\{$ Desires, PB, Conc $\}$ & PlanSet $_{e}$ & $\begin{array}{l}\text { Desires } \\
v \\
\Delta^{J} \\
\end{array}$ \\
\hline $\begin{array}{l}\text { Intention base } \\
\text { Intention }\end{array}$ & $\begin{array}{c}\text { Intentions } \\
\text { Intent } \\
\end{array}$ & Intentions & & $\begin{array}{c}\text { Intentions } \\
8 \\
0 \\
\\
\end{array}$ \\
\hline Plan library & Plans & & & $\underbrace{\text { Plans }}_{\text {PlanSet }}$ \\
\hline Relevant Plans & PlanSet $_{e}$ & $\{$ Plans, Intent, Seq, Cons, L, R $\}$ & $\mathrm{E}_{e}$ & $\mathrm{P}^{\mathrm{P}}$ \\
\hline Plan & Plan & PlanSet $_{e}$ & & $\frac{0}{P B}$ \\
\hline Plan Body & PB & Plan & & $\underbrace{}_{\text {Act }}$ \\
\hline Action & Act & $\{\mathrm{PB}$, Seq, Cons, L, R $\}$ & & Pre \\
\hline Precondition & Pre & $\{$ Act, Plan $\}$ & & $\underbrace{}_{\text {Add }}$ \\
\hline Belief Addition & Add & Act & & $\underset{\text { Del }}{(0)}$ \\
\hline Belief Deletion & Del & Act & & $(0)$ \\
\hline Sequence ; & Seq & $\{\mathrm{PB}$, Try $\}$ & & Try \\
\hline Plan Choice $\triangleright$ & Try & $\{$ Intent, Seq, Goal, L, R $\}$ & & $\frac{5}{\text { Cons }}$ \\
\hline Next Pointer & Cons & $\{$ Seq, Try $\}$ & & (enc \\
\hline Concurrency \| & Conc & $\{$ Seq, Try, PB $\}$ & & 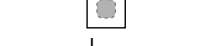 \\
\hline Concurrency Markers & $\{L, R\}$ & Conc & & $($ Goal \\
\hline Declarative Goal & Goal & $\{$ Seq, Try, L, R, PB $\}$ & & $\frac{1}{s c}$ \\
\hline Success Condition & SC & Goal & & $\mathrm{C}_{\mathrm{FC}}$ \\
\hline Failure Condition & FC & Goal & & (3) \\
\hline
\end{tabular}




$$
\begin{aligned}
\llbracket\left\langle\mathcal{B}, E^{e}, \Gamma, \Pi\right\rangle \rrbracket & =\text { Beliefs. } \llbracket \mathcal{B} \rrbracket \| \text { Desires. } \llbracket E^{e} \rrbracket \\
& \| \text { Intentions. } \llbracket \Gamma \rrbracket|| \text { Plans. } \llbracket \Pi \rrbracket \\
\llbracket b_{n} \rrbracket & =\mathrm{B}(n) \\
\llbracket \text { false } \rrbracket & =\text { false } \\
\llbracket \text { true } \rrbracket & =1 \\
\llbracket \mathcal{B}=\left\{b_{1} \ldots b_{n}\right\} \rrbracket & =\llbracket b_{1} \rrbracket|\ldots| \llbracket b_{n} \rrbracket \\
\llbracket E^{e}=\left\{e_{1} \ldots e_{n}\right\} \rrbracket & =\llbracket e_{1} \rrbracket|\ldots| \llbracket e_{n} \rrbracket \\
\llbracket e \rrbracket & =\mathrm{E}_{e} \\
\llbracket \Gamma=\left\{P_{1}, \ldots, P_{n}\right\} \rrbracket & =\text { Intent. } \llbracket P_{1} \rrbracket|\ldots| \text { Intent. } \llbracket P_{n} \rrbracket \\
\llbracket \Pi=\left\{P l_{1} \ldots P l_{n}\right\} \rrbracket & =\prod_{e \in E \text { where } \operatorname{Prigger}\left(P l_{j}\right)=\text { trigger }\left(P l_{k}\right)=e}
\end{aligned}
$$

(a) Beliefs, desire, intention, and plan library encoding.

$$
\begin{aligned}
& \llbracket n i l \rrbracket=1 \\
& \left.\llbracket a c t=\varphi \leftarrow\left\langle\phi^{+}, \phi^{-}\right\rangle \rrbracket=\text { Act.(Pre. } \llbracket \varphi \rrbracket \mid \text { Add. } \llbracket \phi^{+} \rrbracket \mid \text { Del. } \llbracket \phi^{-} \rrbracket\right) \\
& \llbracket \varphi=b_{1} \wedge \cdots \wedge b_{n} \rrbracket=\llbracket b_{1} \rrbracket|\ldots| \llbracket b_{n} \rrbracket \\
& \llbracket \phi^{ \pm}=\left\{b_{1} \ldots b_{n}\right\} \rrbracket=\llbracket b_{1} \rrbracket|\ldots| \llbracket b_{n} \rrbracket \\
& \llbracket P_{1} ; P_{2} \rrbracket=\text { Seq. }\left(\llbracket P_{1} \rrbracket \mid \text { Cons. } \llbracket P_{2} \rrbracket\right) \\
& \left.\llbracket P_{1} \| P_{2} \rrbracket=\text { Conc. (L. } \llbracket P_{1} \rrbracket \mid \mathrm{R} . \llbracket P_{2} \rrbracket\right) \\
& \llbracket \operatorname{goal}\left(\varphi_{s}, P, \varphi_{f}\right) \rrbracket=\text { Goal. }\left(\mathrm{SC} . \llbracket \varphi_{s} \rrbracket|\llbracket P \rrbracket| \mathrm{FC} . \llbracket \varphi_{f} \rrbracket\right) \\
& \llbracket P_{1} \triangleright P_{2} \rrbracket=\text { Try. }\left(\llbracket P_{1} \rrbracket \mid \text { Cons. } \llbracket P_{2} \rrbracket\right) \\
& \llbracket e:\left(\left|\varphi_{1}: P_{1}, \ldots, \varphi_{2}: P_{2}\right|\right) \rrbracket=\text { PlanSet }_{e} \cdot\left(\llbracket \varphi_{1}: P_{1} \rrbracket|\ldots| \llbracket \varphi_{2}: P_{2} \rrbracket\right) \\
& \llbracket \varphi: P \rrbracket=\text { Plan. }(\text { Pre. } \llbracket \varphi \rrbracket \mid \mathrm{PB} . \llbracket P \rrbracket) \\
& \llbracket P l=e: \varphi \leftarrow P \rrbracket=\llbracket \varphi: P \rrbracket
\end{aligned}
$$

(b) Plan and plan-body encoding.

Figure 4: Encoding $\llbracket \cdot \rrbracket$ from BDI agents to bigraphs. 


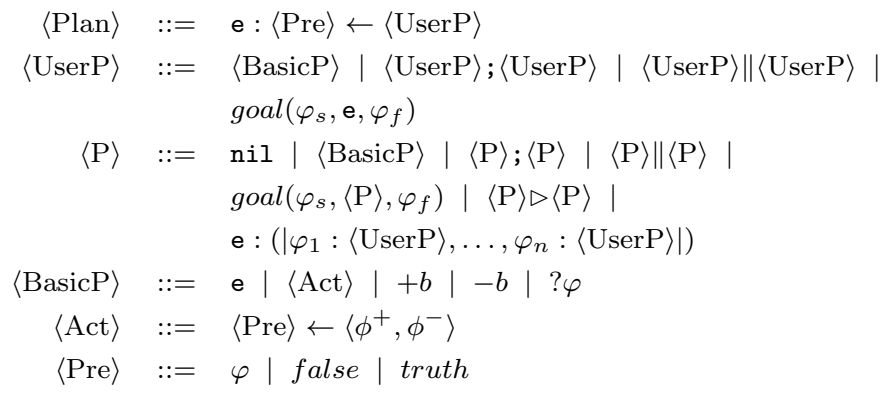

Figure 5: Grammar for Plans and Plan-bodies.

false to represent logical false (rule 4), while the logical constant true is mapped to the empty bigraph (rule 5) as we assume an empty formula is always true, e.g. there may be no pre-condition for some action.

Encoding the belief base $\mathcal{B}$ (and any set concept in general) from a BDI agent to bigraphs leverages the bag-like nature of nesting (rule 6). For empty sets, we have $\llbracket \emptyset \rrbracket=1$, i.e. the bigraph with one empty region.

To encode desires, an entity of $\mathrm{E}_{e}$ is created for each possible event (that an agent desires to respond to) as seen in rules 7 and 8 Importantly, $\mathrm{E}_{e}$ exports a name $e$ that allows us to identify specific events using links. Recall that a set of relevant plans is the set of plans which have the same triggering event. We use this when encoding the plan library $\Pi$ (rule 10) by having it contain sets of relevant plans PlanSet $e_{e}$ with $e$ connecting the event $e$ with the set of plans that respond to it. This differs from typical BDI agents where the plan library is a flat set of plans. This use of indexing by event name through relevant plans decreases the likelihood of some potential human errors, e.g. misspelling of event names and also simplifies agent reasoning by avoiding repetitive searching for relevant plans.

Finally, for intentions, we utilise the same set-like structure as beliefs, this time encoding individual (partially executed) plan-bodies as required (rule 9), which will be discussed in the next section.

\subsection{Encoding Plans and Plan-Bodies}

Plans and plan-bodies are specified with the language given in Fig. 5 which includes includes two forms of plan-bodies: $\langle\mathrm{UserP}\rangle$ that the user writes, and the more comprehensive $\langle\mathrm{P}\rangle$ that can occur during any execution.

A plan e : Pre $\leftarrow\langle\mathrm{UserP}\rangle$ consists of a triggering event $e$, the context (pre-condition) $\langle$ Pre $\rangle$, and a user-defined plan-body specified by $\langle$ UserP $\rangle$. The user-defined plan-body $\langle$ UserP $\rangle$ may be the basic building block $\langle$ BasicP $\rangle$ including handling an internal event $\mathrm{e}$, or executing an action $\langle$ Act $\rangle$. Actions also have the pre-condition $\langle$ Pre $\rangle$, which indicates when an action is valid for execution given in the current belief state. After executing an action, $\phi^{+}$ 
and $\phi^{-}$are sets of beliefs to be added and removed from the belief state, respectively. The user-defined plan-body $\langle\mathrm{UserP}\rangle$ can also be combined in the three ways: $\langle\mathrm{UserP}\rangle ;\langle\mathrm{UserP}\rangle$ executing those two $\langle\mathrm{UserP}\rangle$ in sequence, $\langle\mathrm{UserP}\rangle \|\langle\mathrm{UserP}\rangle$ pursing those two $\langle\mathrm{UserP}\rangle$ concurrently, and $\operatorname{goal}\left(\varphi_{s}, e, \varphi_{f}\right)$ achieving the state $\varphi_{s}$ through addressing an internal event $e$, failing when $\varphi_{f}$ holds, and retrying as long as neither $\varphi_{s}$ nor $\varphi_{f}$ is believed to be true. Internally (i.e. during execution) programs may have an additional three forms: nil is the empty program that is always successful, $\langle\mathrm{P}\rangle \triangleright\langle\mathrm{P}\rangle$ represents trying the first $\langle\mathrm{P}\rangle$ while keeping the second $\langle\mathrm{P}\rangle$ as a backup in case the first $\langle\mathrm{P}\rangle$ fails, and $\mathrm{e}:\left(\left|\varphi_{1}:\langle\mathrm{UserP}\rangle \ldots \varphi_{n}:\langle\mathrm{UserP}\rangle\right|\right)$ is a set of backup plans which are all triggered by the event e.

The bigraph encoding of plans and plan-bodies (Fig. 4b mirrors the grammar given in Fig. 5 by specifying a mapping for each syntactic form. Each individual plan is represented as the pairing of some pre-condition (as encoded belief atoms), nested in the entity Pre, and an encoded plan-body, nested in entity PB (rule 20 and 21] in Fig. 4b).

Bigraphical entities of $\langle\mathrm{UserP}\rangle$ are built by introducing additional controls for each form, e.g. Seq. As the merge product operator of bigraphs is commutative, e.g. $A|B \equiv B| A$, we need to add additional entities to force an ordering on the children. For example, the sequencing $P_{1} ; P_{2}$ (rule 15 in Fig. 4b) utilises an entity Cons that identifies $P_{2}$ as the next to execute after the successful execution of its predecessor $P_{1}$. Likewise, the form $P_{1} \triangleright P_{2}$ (rule 18), that tries $P_{1}$ with $P_{2}$ as a backup, uses Cons to distinguish between $P_{1}$ and $P_{2}$. For concurrency (rule 16) we further require two additional controls L and R to identify the left and right of the concurrency structure $\|$. Finally, for the form of declarative goals $\operatorname{goal}\left(\varphi_{s}, P, \varphi_{f}\right)$ (rule 17), we map it to an entity Goal that nests a success condition SC, failure condition FC and the current form of the remaining program.

Finally, actions are encoded (rule 12) in a similar way. In particular, raw entailment and belief state update forms, i.e. ? $\varphi,+b$, and $-b$, may be seen as special cases of actions that do not update the external environment. We establish the following equivalences to unify them under the same action encoding.

$$
\begin{aligned}
& ? \varphi \equiv \text { act }: \varphi \leftarrow\langle\emptyset, \emptyset\rangle \\
& +b \equiv \text { act }: \emptyset \leftarrow\langle\{b\}, \emptyset\rangle \\
& -b \equiv \text { act }: \emptyset \leftarrow\langle\emptyset,\{b\}\rangle
\end{aligned}
$$

\subsection{Example of Encoding}

To show how our encoding works Table 3 provides the mapping for a BDI agent for the travelling example in Fig. 2.

This completes the structural encoding (i.e. the syntactic specification of a BDI agent), we now turn our attention to a behavioural encoding of BDI agents (i.e. the operation semantics of a BDI agent) as a bigraphical reactive system. We do so in an incremental manner in the following three steps: in Section 4 we define the semantics of a subset of CAN that we call the core CAN. Core 
Table 3: Example encoding of a conference travel agent in Fig. 2

\begin{tabular}{|c|c|}
\hline Agent & $\llbracket$ Agent $\rrbracket$ \\
\hline $\mathcal{B}=\left\{b_{1}, b_{2}, b_{6}, b_{7}\right\}$ & Beliefs. $(B(1)|B(2)| B(6) \mid B(7))$ \\
\hline$\Pi=\left\{P l_{1}, P l_{2}, P l_{3}\right\}$ & Plans. (PlanSet $e_{1} \cdot\left(\llbracket P l_{1} \rrbracket \mid \llbracket P l_{2} \rrbracket\right) \mid$ PlanSet $\left._{e_{2}} \cdot \llbracket P l_{3} \rrbracket\right)$ \\
\hline$P l_{1}=e_{1}: \varphi_{1} \leftarrow a c t_{1} ; a_{c} t_{2}$ & 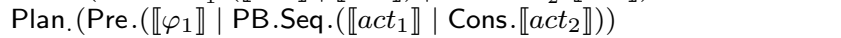 \\
\hline$P l_{2}=e_{1}: \varphi_{2} \leftarrow a c t_{3} ; e_{2} ; a c t_{4}$ & 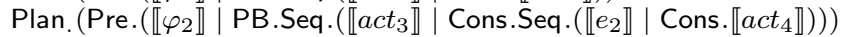 \\
\hline $\begin{array}{l}P l_{3}=e_{2}: \varphi_{3} \leftarrow a{ }_{c} ; a c t_{6} \\
\varphi_{1}=b_{1} \wedge b_{2}\end{array}$ & $\begin{array}{l}\text { Plan. (Pre. }\left(\llbracket \varphi_{3} \rrbracket \mid \text { PB.Seq. }\left(\llbracket a c t_{5} \rrbracket \mid \text { Cons. } \llbracket a c t_{6} \rrbracket\right)\right) \\
\mathrm{B}(1) \mid \mathrm{B}(2)\end{array}$ \\
\hline$a c t_{1}=b_{3} \leftarrow\left\langle\left\{b_{4}\right\}, \emptyset\right\rangle$ & Act.(Pre.B(3)|Add.B(4)|Del.1) \\
\hline$a^{a c t_{2}}=b_{4} \leftarrow\left\langle\left\{b_{5}\right\}, \emptyset\right\rangle$ & Act.(Pre.B(4) |Add.B(5)|Del.1) \\
\hline$\varphi_{2}=b_{6} \wedge b_{7}$ & $\mathrm{~B}(6) \mid \mathrm{B}(7)$ \\
\hline act $_{3}=$ true $\leftarrow\left\langle\left\{b_{8}\right\}, \emptyset\right\rangle$ & Act.(Pre.1 | Add.B(8)| Del.1) \\
\hline$e_{2}$ & $\mathrm{E}_{e_{2}}$ \\
\hline$\left.a c t_{4}=b_{9} \leftarrow\left\langle\left\{b_{5}\right\}, \emptyset\right\}\right\rangle$ & Act.(Pre.B(9)|Add.B(5)|Del.1) \\
\hline$\varphi_{3}=b_{8}$ & $\mathrm{~B}(8)$ \\
\hline act $_{5}=b_{8} \leftarrow\left\langle\left\{b_{10}\right\}, \emptyset\right\rangle$ & Act.(Pre.B(8)|Add.B(10)|Del.1) \\
\hline act $_{6}=b_{10} \leftarrow\left\langle\left\{b_{9}\right\},\left\{b_{8}, b_{10}\right\}\right\rangle$ & Act.(Pre.B(10)|Add.B(9)|Del.(B(8)|B(10))) \\
\hline
\end{tabular}

CAN semantics excludes concurrency and declarative goals, and so resembles AgentSpeak [2]. In Section 5] we encode core CAN as a BRS and in Section 6 we extend such a BRS for the core CAN to include concurrency and declarative goals.

\section{Semantics of Core CAN Language}

\subsection{Overview of Core CAN language}

The core operation of an agent in response to an (external) event is as follows. All relevant plans for that event are retrieved from the (pre-defined) plan library. An applicable plan is selected (if one exists) and its plan-body is added to the intention base. The plan-body consists of discrete steps, e.g. actions or subevents. When executing a sub-event, its applicable plan requires to be found, and its plan-body is also added to the intention base - this forms an execution tree within the intention. A BDI agent continues to execute until there are no pending events, and all intentions are completed (either successfully or with failure).

\subsection{Core CAN Semantics}

We specify the behaviour of an agent as an operational semantics 24] defined over configurations $\mathcal{C}$ and transitions $\mathcal{C} \rightarrow \mathcal{C}^{\prime}$. Transitions $\mathcal{C} \rightarrow \mathcal{C}^{\prime}$ denote a single execution step between configuration $\mathcal{C}$ and $\mathcal{C}^{\prime}$. We write $\mathcal{C} \rightarrow($ resp. $\mathcal{C} \nrightarrow)$ to state that there is (resp. is not) a $\mathcal{C}^{\prime}$ such that $\mathcal{C} \rightarrow \mathcal{C}^{\prime}$.

A derivation rule specifies the necessary conditions for an an agent to transition to a new configuration. A derivation rule consists of a (possibly empty) set of premises $p_{i}(i=1, \ldots, n)$ on $\mathcal{C}$, and a conclusion, denoted by

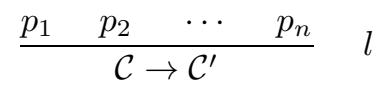




$$
\begin{aligned}
& \frac{a c t: \psi \leftarrow\left\langle\phi^{-}, \phi^{+}\right\rangle \quad \mathcal{B} \vDash \psi}{\langle\mathcal{B}, a c t\rangle \rightarrow\left\langle\left(\mathcal{B} \backslash \phi^{-} \cup \phi^{+}\right), n i l\right\rangle} \text { act } \quad \frac{\mathcal{B} \models \phi}{\langle\mathcal{B}, ? \phi\rangle \rightarrow\langle\mathcal{B}, \text { nil }\rangle} ? \\
& \overline{\langle\mathcal{B},+b\rangle \rightarrow\langle\mathcal{B} \cup\{b\}, n i l\rangle}+b \quad \overline{\langle\mathcal{B},-b\rangle \rightarrow\langle\mathcal{B} \backslash\{b\}, n i l\rangle}-b \\
& \frac{\Delta=\left\{\varphi: P \mid\left(e^{\prime}=\varphi \leftarrow P\right) \in \Pi \wedge e^{\prime}=e\right\}}{\langle\mathcal{B}, e\rangle \rightarrow\langle\mathcal{B}, e:(|\Delta|)\rangle} \text { event } \\
& \frac{\varphi: P \in \Delta \quad \mathcal{B} \models \varphi}{\langle\mathcal{B}, e:(|\Delta|)\rangle \rightarrow\langle\mathcal{B}, P \triangleright e:(|\Delta \backslash\{\varphi: P\}|)\rangle} \text { select } \\
& \frac{\left\langle\mathcal{B}, P_{1}\right\rangle \rightarrow\left\langle\mathcal{B}^{\prime}, P_{1}^{\prime}\right\rangle}{\left.\left\langle\mathcal{B}, P_{1} \triangleright P_{2}\right\rangle \rightarrow\left\langle\mathcal{B}^{\prime}, P_{1}^{\prime} \triangleright P_{2}\right)\right\rangle} \triangleright_{;} \quad \frac{}{\left\langle\mathcal{B},\left(\text { nil } \triangleright P_{2}\right)\right\rangle \rightarrow\left\langle\mathcal{B}^{\prime}, \text { nil }\right\rangle} \triangleright_{\top} \\
& \frac{P_{1} \neq \text { nil }\left\langle\mathcal{B}, P_{1}\right\rangle \nrightarrow \quad\left\langle\mathcal{B}, P_{2}\right\rangle \rightarrow\left\langle\mathcal{B}^{\prime}, P_{2}^{\prime}\right\rangle}{\left\langle\mathcal{B}, P_{1} \triangleright P_{2}\right\rangle \rightarrow\left\langle\mathcal{B}^{\prime}, P_{2}^{\prime}\right\rangle} \triangleright_{\perp} \\
& \frac{\left\langle\mathcal{B}, P_{1}\right\rangle \rightarrow\left\langle\mathcal{B}^{\prime}, P_{1}^{\prime}\right\rangle}{\left\langle\mathcal{B},\left(P_{1} ; P_{2}\right)\right\rangle \rightarrow\left\langle\mathcal{B}^{\prime},\left(P_{1}^{\prime} ; P_{2}\right)\right\rangle} ; \quad \frac{\langle\mathcal{B}, P\rangle \rightarrow\left\langle\mathcal{B}^{\prime}, P^{\prime}\right\rangle}{\langle\mathcal{B},(n i l ; P)\rangle \rightarrow\left\langle\mathcal{B}^{\prime}, P^{\prime}\right\rangle} ; \top
\end{aligned}
$$

Figure 6: Core CAN semantics.

where $l$ is a rule name. We write $\mathcal{C} \stackrel{l}{\rightarrow} \mathcal{C}^{\prime}$ to denote $\mathcal{C}$ evolves to $\mathcal{C}^{\prime}$ through the application of derivation rule $l$.

The CAN semantics were originally defined [ [6] over the triple $\langle\mathcal{B}, \mathcal{A}, P\rangle$ where $\mathcal{B}$ is the current belief base, $\mathcal{A}$ the sequence of actions that have been executed, and $P$ the current partially executed plan-body. As the recorded sequence of executed actions is never used to determine the operation of an agent, i.e. there are no pre-condition on $\mathcal{A}$, we do not include it here (i.e. $\langle\mathcal{B}, P\rangle$ ). It is trivial to $\log$ the action sequence within the bigraph model if required, however we do not do so here because an action log introduces states that would otherwise be isomorphic (resulting in larger transition systems).

The semantics of CAN language is specified by two types of transitions. The first transition type, denoted as $\rightarrow$, specifies intention-level evolution in terms of configuration $\langle\mathcal{B}, P\rangle$ where $\mathcal{B}$ is the current belief set, and $P$ the plan-body currently being executed (i.e. the next step of the current intention). The second type, denoted as $\Rightarrow$, specifies agent-level evolution over $\left\langle E^{e}, \mathcal{B}, \Gamma\right\rangle$, detailing how to execute a complete agent where $E^{e}$ stands for the a set of pending external events required to address.

Fig. 6 gives the set of derivation rules for evolving any single intention. For example, derivation rule act handles the execution of an action, when the precondition is met, resulting in a belief state update. Rules ?, $+b$ and $-b$ are special actions that perform pre-condition check (?), adding one belief atom $(+b)$ and deleting atoms $(-b)$. As in Section 3 we assume an equivalence between $a c t$ and $?,+b,-b$ and do not directly model these rules. Rule event replaces an event with the set of relevant plans, while rule select chooses an applicable plan from a set of relevant plans while retaining un-selected plans as backups. With these backup plans, the rules for failure recovery $\triangleright_{;}, \triangleright_{T}$, and $\triangleright_{\perp}$ enable 


$$
\begin{gathered}
\frac{e \in E^{e}}{\left\langle E^{e}, \mathcal{B}, \Gamma\right\rangle \Rightarrow\left\langle E^{e} \backslash\{e\}, \mathcal{B}, \Gamma \cup\{e\}\right\rangle} A_{\text {event }} \\
\frac{P \in \Gamma \quad\langle\mathcal{B}, P\rangle \rightarrow\left\langle\mathcal{B}^{\prime}, P^{\prime}\right\rangle}{\left\langle E^{e}, \mathcal{B}, \Gamma\right\rangle \Rightarrow\left\langle E^{e}, \mathcal{B}^{\prime},(\Gamma \backslash\{P\}) \cup\left\{P^{\prime}\right\}\right\rangle} A_{\text {step }} \\
\frac{P \in \Gamma \quad\langle\mathcal{B}, P\rangle \nrightarrow}{\left\langle E^{e}, \mathcal{B}, \Gamma\right\rangle \Rightarrow\left\langle E^{e}, \mathcal{B}, \Gamma \backslash\{P\}\right\rangle} A_{\text {update }}
\end{gathered}
$$

Figure 7: Derivation rules for agent configuration.

new plans to be selected if the current plan fails (due to e.g. the unexpected environment changes). Finally, rules ; and $; \top$ describe executing plan-bodies in sequence.

The agent-level semantics are given in Fig. 7. An agent configuration is defined by the triple $\left\langle E^{e}, \mathcal{B}, \Gamma\right\rangle$ consisting of a set of external events $E^{e}$ to which the agent is required to respond, the belief set $\mathcal{B}$, and the intention base $\Gamma-\mathrm{a}$ set of partially executed plan-bodies $P$ that the agent has already committed to. The derivation rule $A_{\text {event }}$ handles external events, which originate from the environment 4 , by adopting them as intentions. Rule $A_{\text {step }}$ selects an intention from the intention base, and evolves a single step w.r.t. intention-level transition, while $A_{\text {update }}$ discards intentions which cannot make any intention-level transition (either because it has already succeeded, or it failed) 5 .

\subsection{Example of Core CAN Semantics}

To show how an agent evolves in the CAN semantics we use the conference travelling example in Fig. 2. Assuming the external event $e_{1}$ has already been converted from a desire to an intention, Fig. 8 illustrates the intention-level evolution of this intention according to the rules presented in Fig. 6 . In Fig. 8 agents evolve from left to right, each line consists of a single step of an intention. Below each step we show the sub-rules that applied. A commentary is as follows.

When the event $e_{1}$ is posted to the agent, the event rule in Fig. 6 transforms $e_{1}$ into the program containing all the relevant plans available (1). If the agent believes that it owns a car and the venue is within driving distance (i.e. $\varphi_{1}$ holds holds), then the select rule transforms the set of relevant plans into the selected plan (2), which indicates the sequence $a c t_{1} ; a c t_{2}$ is ready for execution, while the other plans are indicated as backup on the right-hand side of the symbol $\triangleright$. Next, the agent tries to execute the program act $_{1} ; a_{c} t_{2}$. Given the belief base in Fig. 2 the pre-condition $a c t_{1}$ does not hold (e.g. the car engine fails to start), thus act $\nrightarrow$. Meanwhile, the backup plan is applicable shown by

\footnotetext{
${ }^{4}$ As we do not model the environment explicitly, we assume any events are waiting in the desire set at the start of an agent execution.

${ }^{5}$ In the original CAN semantics there is no way to determine if an event was handled successfully or not, both cases are treated the same way (by removing the intention when it is done or cannot progress).
} 


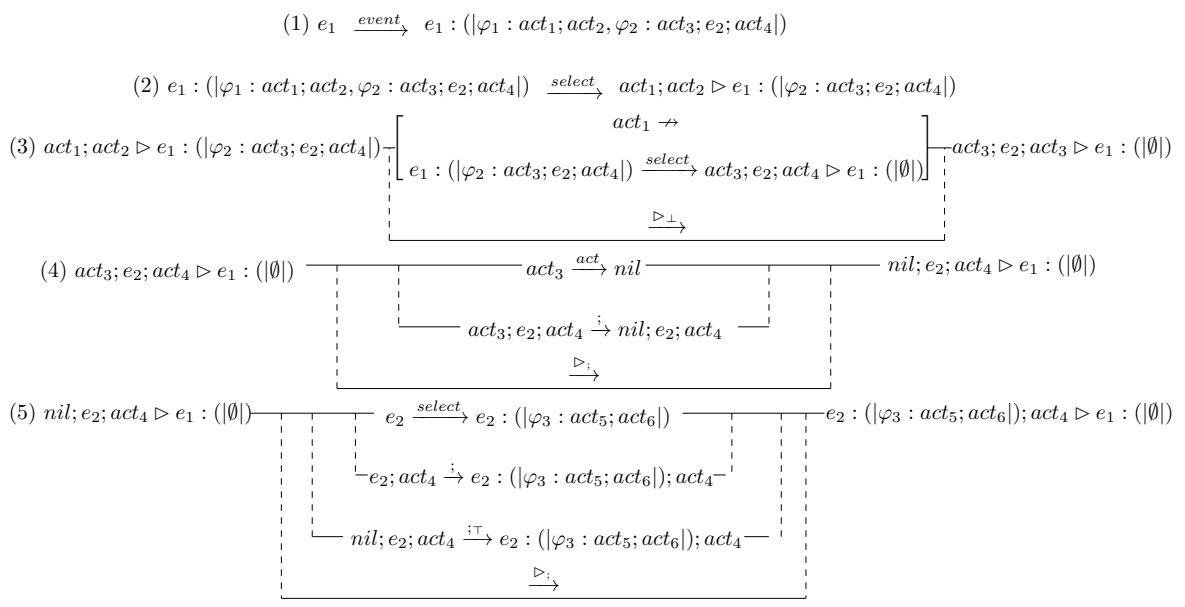

Figure 8: Illustration of intention-level evolution of the event $e_{1}$.

the derivation select from $e_{1}:\left(\mid \varphi_{2}:\right.$ act $_{3} ; e_{2} ;$ act $\left._{4} \mid\right)$ to act act $_{3} ; e_{2} ;$ act $_{4} \triangleright e_{1}:(|\emptyset|)$. According to the rule $\nabla_{\perp}$, the agent can initiate the failure recovery by trying such a backup plan, resulting in the program shown in (3). Since act $_{3}$ has true as its pre-condition, it can always be executed shown by act ${ }_{3} \stackrel{\text { act }}{\longrightarrow}$ nil. After execution of $a c t_{3}$, the rule ; then updates the entire sequence from act $_{3} ; e_{2} ; a$ act $_{4}$ to $n i l ; e_{2} ; a c t_{4}$. After the left-hand side of $\triangleright$ is updated, the rule $\triangleright$; can then further transform the program to that in (4). In order to discard the symbol nil in a sequence, it requires the part after nil in a sequence to be progressed, namely $e_{2} ; a c t_{4}$. To progress the $e_{2} ; a c t_{4}$, it requires to progress the first part of such a sequence, i.e. $e_{2}$. To progress the event $e_{2}$, it requires to retrieve a set of its relevant plans. Therefore, we have what is shown in the (5). The rule select firstly transforms the event $e_{2}$ to a set of relevant plans, secondly the rule ; updates the sequence $e_{2} ; a c t_{4}$, and thirdly the symbol nil can be removed by the rule $; \top$ from the entire sequence $n i l ; e_{2} ; a c t_{4}$. Finally, the rule $\triangleright$; can follow up transforming the entire program on the left-hand side of $\triangleright$ accordingly.

For brevity, we omit the rest of the evolution. In practice an agent may execute multiple intentions concurrently.

\subsection{AND/OR Trees}

We can view the semantic evolution of the agent program in terms of reductions over $\mathrm{AND} / \mathrm{OR}$ trees, and use this representation to reason about the interactions between events, plans, and intentions [25, 26]. AND nodes are successful if all of their children succeed while OR nodes are successful if at least one child succeeds. We make heavy use of such AND/OR trees in our behavioural encoding of CAN semantics in bigraphs that can be seen (in part) as reductions over these trees. However, we stress that although the behaviour of a CAN agent can be visualised via AND/OR trees, in practice, the trees are not fully realised in memory and are created on-demand as the intention evolves. 
The root of an AND/OR tree is a top-level external event represented as an OR node, that is, an event succeeds if at least one plan succeeds. The tree is built implicitly through the syntax of CAN. For example, the sequencing symbol ; ensures that execution must successfully execute all steps in the planbody to allow the parent AND node to succeed. Meanwhile, the failure recovery symbol $\triangleright$ represents choice, with backup plans creating the branching structure. In Section 6.0.1, an additional form $\|$ will be introduced to complement ; by identifying branches that can be explored concurrently.

As an example we revisit the conference travelling example of Fig. 2 showing one possible AND/OR tree for the plans $P l_{1}=e_{1}: \varphi_{1} \leftarrow$ act $t_{1} ; a c t_{2}, P l_{2}=e_{1}$ : $\varphi_{2} \leftarrow a c t_{3} ; e_{2} ; a c t_{4}$, and $P l_{3}=e_{2}: \varphi_{3} \leftarrow a c t_{5} ; a c t_{6}$. In this case, $P l_{1}$ was chosen first and $\mathrm{Pl}_{2}$ kept as a backup plan as shown in Fig. 9. The top-level event $e_{1}$ is achieved if either of the two plans $P l_{1}$ or $P l_{2}$ are successful. In this case the agent has chosen to do $P l_{1}$ before $P l_{2}$, although the ordering is not fixed ahead of execution time. The plan $P l_{1}$ itself involves performing the actions $a c t_{1}$ followed by act 2 , whereas one part of plan-body of plan $\mathrm{Pl}_{2}$ involves achieving the sub-event $e_{2}$ which can, in turn, be addressed by the plan $\mathrm{Pl}_{3}$.

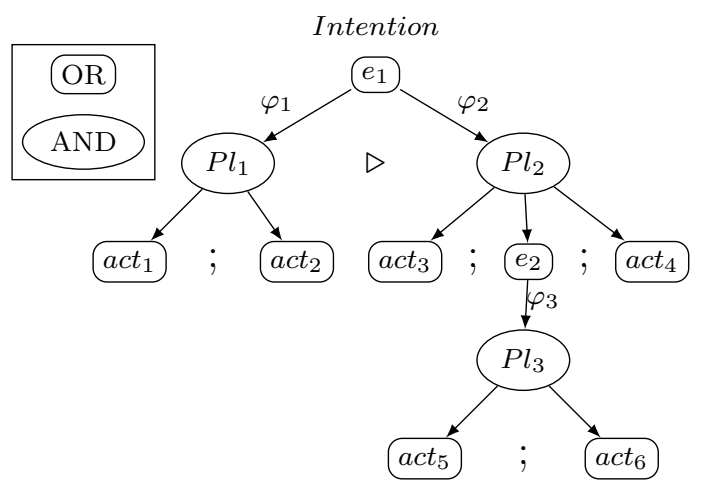

Figure 9: Snapshot of $\mathrm{AND} / \mathrm{OR}$ tree representing the intention for the event $e_{1}$ where the agent chose to try $P l_{1}$ before $P l_{2}$ during execution.

From the point of view of the semantics, the tree is explored in a depthfirst manner with reductions being pushed down the tree. For example, the derivation rule ; reduces a given branch of the tree while the rule ; $T$ moves to the next child at the same AND level. When a node cannot be reduced, e.g. if an action pre-condition is unmet, this failure propagates to the closest branch point (OR-node) where they are handled by the failure recovery rules (e.g. $\left.\triangleright_{\perp}\right)$.

\section{Encoding Core Can Semantics in Bigraphs}

We now encode the core CAN semantics (presented in Figs. 66 and 7) as a bigraphical reactive system (BRS) and show that the encoding is faithful. By faithful we mean that for each transition $\stackrel{l}{\Rightarrow}$ (resp. intention $\stackrel{l}{\rightarrow})\left\langle E^{e}, \mathcal{B}, \Gamma\right\rangle \stackrel{l}{\Rightarrow}$ 
Table 4: Additional entities for semantics encoding

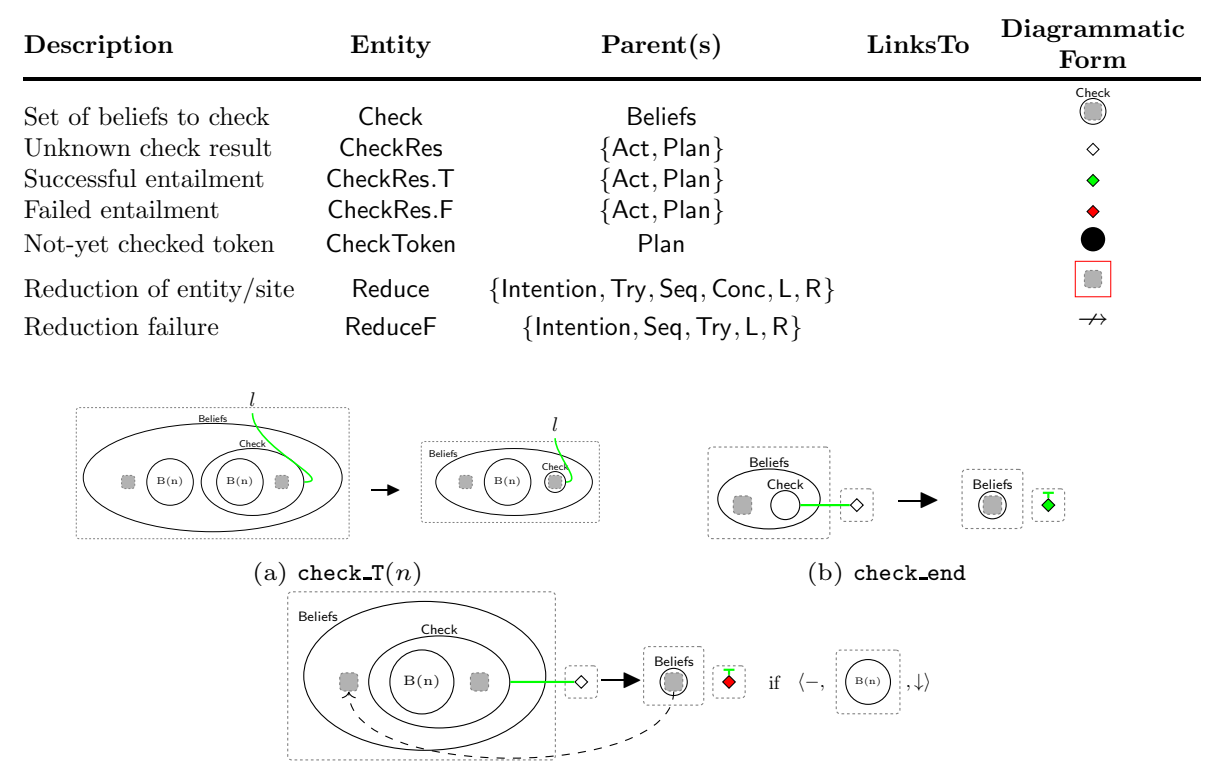

(c) check_F

Figure 10: Reactions for logical entailment.

$\left\langle E^{\prime e}, \mathcal{B}^{\prime}, \Gamma^{\prime}\right\rangle\left(\right.$ resp. $\left.\langle\mathcal{B}, P\rangle \stackrel{l}{\Rightarrow}\left\langle\mathcal{B}^{\prime}, P^{\prime}\right\rangle\right)$ there exists a finite sequence of reaction rules, such that $\llbracket\left\langle E^{e}, \mathcal{B}, \Gamma\right\rangle \rrbracket \rightarrow \rightarrow^{+} \llbracket\left\langle E^{\prime e}, \mathcal{B}^{\prime}, \Gamma^{\prime}\right\rangle \rrbracket$ (resp. $\left.\llbracket\langle\mathcal{B}, P\rangle \rrbracket \rightarrow \longrightarrow^{+} \llbracket\left\langle\mathcal{B}^{\prime}, P^{\prime}\right\rangle \rrbracket\right)$ and no derivation rules in BDI side is enabled that were not available in the initial state. The encoding may introduce new intermediate states, but they do not enable additional BDI derivation rules, i.e. there is no additional branching.

To encode control flow required for execution, we require additional entities that are not part of the structural encoding, i.e. they do not necessarily have a corresponding agent representation in CAN. These additional entities are given in Table 4 and their purpose is introduced as they are used.

For brevity, we give an overview of key aspects of the encoding. The full executable model, for use with BigraphER [19], is available [27].

\subsection{Belief Checks and Updates}

The CAN semantics assumes set operations and logic entailment as builtin operators. However, as we want an executable semantics, these must be explicitly encoded in the BRS.

We encode belief updates and checks in the usual recursive manner as shown in Fig. 10. For example, the reaction rule check_end provides a base-case for check_T $(n)$, while check_F (a conditional rule) handles the case when there is no match in the belief base. Similar reaction rules (not shown) are provided to 
perform addition and deletion of belief: 6 .

The belief check reaction rules use auxiliary entities, e.g. Check $l$ and CheckRes $l$ (shown as a diamond). These auxiliary entities, which are added from other reaction rules, encode control flow. As these entities are not part of the CAN syntax encoding, they do not enable any additional agent steps. After performing the sequence of reaction rules equivalent to a CAN derivation rule, no auxiliary entities will be present - they are only allowed in intermediate states.

Notice the number of children of Check decreases on each reaction rule application suffices to prove that the logical entailment (resp. checks/updates) will complete in a finite number of steps. As such, placing belief checks/updates into the highest rule priority class of the BRS allows us to assume belief checks/updates are atomic with respect to the other reactions. That is, an agent never sees a part-modified belief set (as required to model atomic actions).

We use the label

$$
\begin{aligned}
\{\text { set_ops }\}= & \text { check_T }(n), \text { check_end, check_F, } \\
& \text { del_in }(n), \text { del_notin }(n), \text { delete_end, } \\
& \text { add_end, add_notin }(n), \text { add_in }(n)
\end{aligned}
$$

to refer to the priority class of set operations.

\subsection{Modelling Reductions}

The CAN semantics assumes a notion of irreducibility. That is, the derivation rule $\langle\mathcal{B}, P\rangle \nrightarrow$ represents the failure of an agent to perform any further operation on the program $P$. For example, $\langle\mathcal{B}$, act $\rangle \nrightarrow$ holds if the pre-condition of the action act is not met.

While CAN remains agnostic to such details, we require the notion of irreducibility to be explicitly encoded to obtain an executable semantics. To encode explicit reduction, we introduce auxiliary controls Reduce (by colouring the entity/site being reduced as red) and ReduceF (representing $\nrightarrow$ ).

Reduce requests the entities nested below are reduced, for example by executing an action. In the case reduction is not possible, e.g. if an action precondition is not met, ReduceF represents the failure to reduce, enabling checks of the premise $\langle\mathcal{B}, P\rangle \nrightarrow$ in derivation rules.

If we view intentions as $\mathrm{AND} / \mathrm{OR}$ trees, the explicit reductions perform the tree search with Reduce determining which sub-tree to reduce next, and ReduceF indicating a sub-tree could not reduce and backtracking should be performed.

We define a function $\llbracket \mathbb{\|}:\langle\mathcal{B}, P\rangle \rightarrow \mathbf{B g}(\mathcal{K} \cup$ Reduce $)$ that, for belief base $\mathcal{B}$ and intention-level program $P$, requests that the sub-tree rooted at $P$ be reduced. That is:

$$
\llbracket\langle\mathcal{B}, P\rangle \rrbracket \stackrel{\text { def }}{=} \llbracket \mathcal{B} \rrbracket \| \text { Reduce. } \llbracket P \rrbracket
$$

\footnotetext{
${ }^{6}$ We assume additions/deletions are disjoint (as they are in practice) so that there are no race conditions between the reactions.
} 


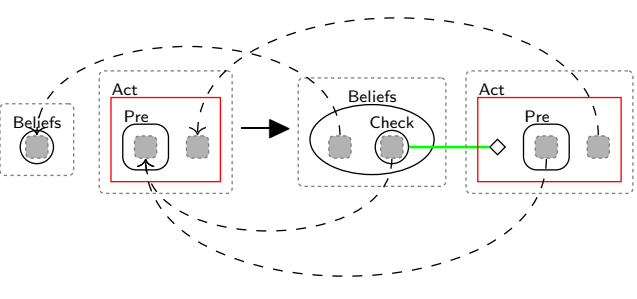

(a) act_check

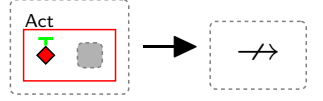

(b) act_F

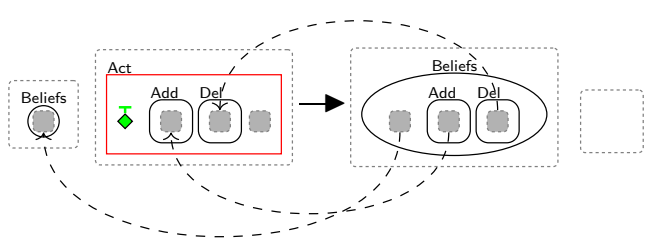

(c) act_T

Figure 11: Reactions for actions.

Where $\mathcal{B}$ is a mutable, globally scoped, environment for the reduction of $P$. This is key benefit of bigraphs for modelling: environments can be placed in parallel.

The function $\llbracket \rrbracket$ for reduction plays a key role in our semantic encoding. For example, it forms the bridge between agent-level steps and intention-level steps, i.e. an agent $\left\langle\mathcal{B}, E^{e},\{P \cup \Gamma\}, \Pi\right\rangle$ can (try to) step intention $P$ using $\mathbb{\llbracket}\langle\mathcal{B}, P\rangle \rrbracket$.

\subsection{Core Semantic Encoding}

Given the atomic set operations and explicit reduction, We now show how the core CAN semantics are encoded as a BRS.

\subsubsection{Actions}

The main operation of an agent is to execute actions that update both the external environment, e.g. moving a block, and in-turn revise the internal belief base. Recall that, in the encoding of syntax of CAN language, we have established entailment and belief state updates (rules $? \varphi,+b,-b$ ) as special cases of actions that simply do not update the external environment. As such, we can safely omit the explicit reactions for entailment and belief state updates.

If the pre-condition of an action is true, i.e. $\mathcal{B} \models \phi$, performing (or reducing) an action consists of the reactions act_check and act_T as shown in Fig. 11 Firstly, the reaction act_check requests the action pre-condition to be checked by nesting a Check entity within the belief base. As we have established set operations to be the highest priority class, we know a belief check operation is finite and applies atomically. Therefore, it does not alter the shape of $\llbracket \mathcal{B} \rrbracket$ (i.e. no other CAN rules are enabled). After successful entailment of the action 
pre-condition, the reaction rule act_T performs the action by updating the belief base. Once again, given the priority of set operations, the set updates will be effectively atomic and no other CAN derivation rule can interrupt such a belief update.

Lemma 1. (Faithfulness of act) When act is applicable it has corresponding finite reaction sequence $\mathbb{\|}\left\langle\mathcal{B}\right.$, act $\left.: \varphi \leftarrow\left\langle\phi^{+}, \phi^{-}\right\rangle\right\rangle \rrbracket \rightarrow \rightarrow^{+} \llbracket\left\langle\mathcal{B}^{\prime}\right.$, nil $\rangle \rrbracket$.

Proof. $\rightarrow+$ contains only reaction rules

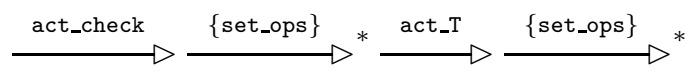

where all rules are finite, set_ops applies atomically, and, by only adding auxiliary variables, all rules do not introduce intermediate states that add new branching points (as required).

We have discussed what happens when the pre-condition of an action holds. However, it is not explicit in CAN semantics what should be done in the case the pre-condition check fails. From the perspective of an AND/OR tree, as actions are always under AND nodes, the failure needs to be propagated upwards in order to enable failure recovery to take place. As introduced in Section 5.2, the entity ReduceF is provided to explicitly denote the reduction failure. Therefore, we can have the reaction act $F$ to report the failure depicted in Fig. 11b. Reducing to ReduceF enables checking of the premise $\langle B, a c t\rangle \nrightarrow$ (as is done implicitly in CAN semantics). Once a failure is reported, other reaction can be triggered to, for example, recover from the failure.

\subsubsection{Plan Selection}

Recall that the agent responds to an event by selecting an applicable plan from a set of pre-defined plans. The following two derivation rules specify the plan selection. The first rule event converts an event to the set of plans that respond to that event (i.e. relevant plans), while the second rule select chooses an applicable plan (if exists) from the set of relevant plans.

The reaction rule corresponding to the derivation rule event is depicted in Fig. 12. As the syntax encoding uses links to connect an event $\mathrm{E}_{e}$ to its set of relevant plans $\mathrm{PlanSet}_{e}$, we can encode the derivation rule event with a single reaction rule by replacing the event entity $\mathrm{E}_{e}$ with PlanSet $_{e}$ as shown in Fig. 12 .

Lemma 2. (Faithfulness of event) event has a corresponding finite reaction sequence $\llbracket\langle\mathcal{B}, e\rangle \rrbracket \rightarrow+\llbracket\langle\mathcal{B}, e:(|\Delta|)\rangle \rrbracket$.

Proof. $\rightarrow+$ contains only $\stackrel{\text { reduce_event }}{\longrightarrow}$. Trivial.

The derivation rule select is modelled in a similar style to how to execute an action, beginning with the pre-condition check against the belief base before selecting an appropriate plan (if one exists). In detail, the reaction rule select_plan_check (in Fig. 13a) firstly finds a plan that has not yet had the 


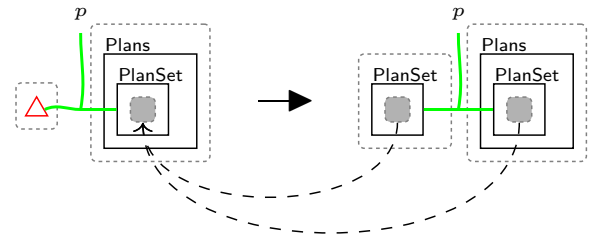

Figure 12: reduce_event

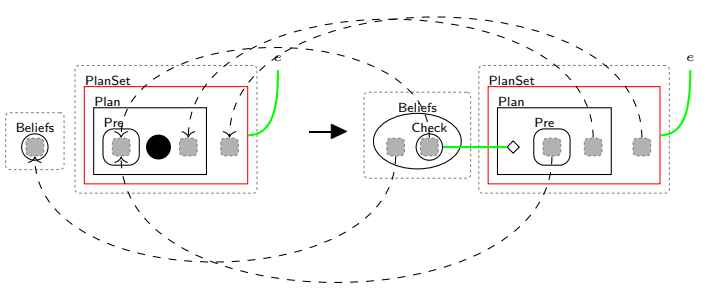

(a) select_plan_check

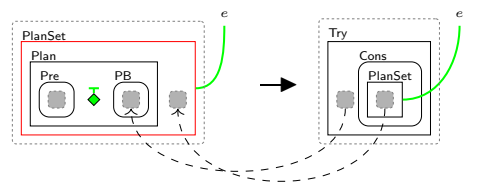

(b) select_plan_T

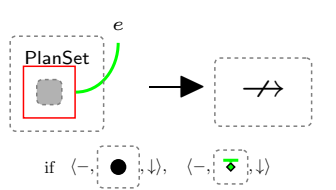

(c) select_plan_F

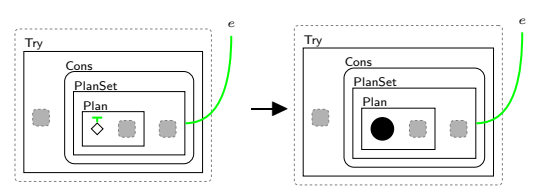

(d) reset_planset

Figure 13: Reactions for plan selection. 
pre-conditions checked - facilitated via an automatically-added auxiliary entity CheckToken that records if a plan has already been considered - and initiates an operation to check the plan pre-condition. To ensure the automatic addition of the entity CheckToken to all Plan entities within the Plans perspective, an additional reaction rule is executed once at the start of a model execution to update the plan library. As this is an implementation detail we do not add the tokens directly to the syntax encoding in the section Section 3 After checking the pre-condition of a plan is true, the reaction rule select_plan_T (in Fig. 13b removes the selected applicable plan from the set of relevant plans, and converts it into $\triangleright$ form and keeping the rest of plans as backups.

Lemma 3. (Faithfulness of select) When the set of relevant plans $(|\Delta|$ ) is non-empty and contains at least one applicable plan for a given event, the CAN derivation rule select has a corresponding finite reaction sequence $\mathbb{\amalg}\langle\mathcal{B}, e$ : $(|\Delta|)\rangle \rrbracket \rightarrow+\llbracket\langle\mathcal{B}, P \triangleright e:(\Delta \backslash\{\varphi: P\})\rangle \rrbracket$.

Proof. Finiteness of the plan pre-condition entailment and the use of CheckToken auxiliary controls ensures an applicable plan to be found in a finite number of reactions with $\longrightarrow+$ containing $\stackrel{\text { select_plan_check }}{\longrightarrow}{ }^{*} \stackrel{\text { select_plan_T }}{\longrightarrow}$. As the reaction select_plan_check only uses auxiliary controls, and select_plan_T performs the intention update in a single step, no new CAN derivation rules are enabled.

If no plan is applicable (a failure), the reaction rule select_plan_F (in Fig. 13c) propagates a ReduceF up the tree. We use a conditional rule to ensure the plan selection only fails if all plans have been checked (or there are no plans), i.e. when there are no CheckToken entities left, and no plan that was checked is applicable. Finally, an auxiliary reaction rule reset_planset (Fig. 13d) ensures that after plan selection, the remaining unchosen (but checked) plans are re-assigned the control CheckToken to allow the plan to be checked again if failure recovery is required.

\subsubsection{Tree Reductions}

The remaining CAN intention-level derivation rules specify how the AND/OR tree should be explored. For example that in the sequencing structure $P_{1} ; P_{2}$ we should reduce $P_{1}$ before $P_{2}$.

The derivation rules; and ; $T$ describe how to progress the sequencing of $P_{1} ; P_{2}$. The derivation rule; is encoded by the reaction rule reduce_seq (Fig. 14a) that pushes reduction into the first child of a sequence. The use of a site (i.e. an abstraction) in bigraphs allows this single rule to handle any type of program $P$.

Lemma 4. (Faithfulness of;); has a corresponding finite reaction sequence $\llbracket\left\langle\mathcal{B}, P_{1} ; P_{2}\right\rangle \rrbracket \rightarrow+\llbracket\left\langle\mathcal{B}^{\prime}, P_{1}^{\prime} ; P_{2}\right\rangle \rrbracket$.

Proof. Structural induction on $\llbracket \mathcal{B}, P_{1} \rrbracket$. The specific form of $\rightarrow+$ depends on the program $P_{1}$ (e.g. act). However, as the AND/OR tree is finite, the reductions under $P_{1}$ are also finite. No reductions occur under Cons as required. 


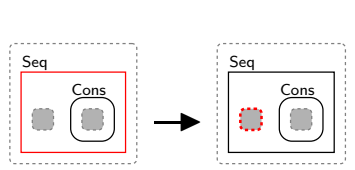

(a) reduce_seq

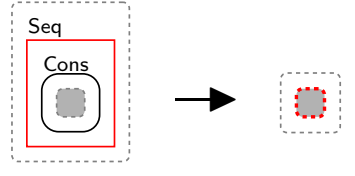

(b) seq_succ

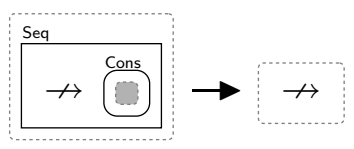

(c) seq_fail

Figure 14: Reactions for sequencing with priorities: reduce_seq $<\{$ seq_succ, sec_fail $\}$

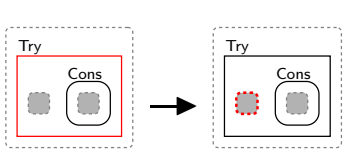

(a) try_seq

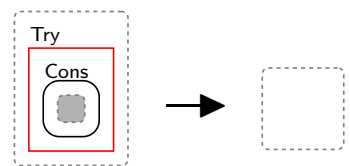

(b) try_succ

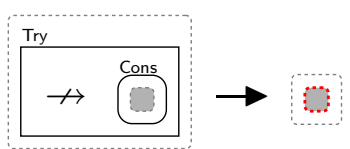

(c) try_failure

Figure 15: Reactions for recovery with priorities: try_seq $<\{$ try_succ,try_failure $\}$.

The derivation rule ; $T$ is encoded using the reaction rule seq_succ (Fig. 14b) that matches in the case the first part of the sequence completed successfully, i.e. $\llbracket n i l \rrbracket=1$. As specified in the derivation rule in CAN, we not only make the children under Cons the new current program, but we also (try to) reduce it immediately.

Lemma 5. (Faithfulness of $; \top$ ) ; $\uparrow$ has a corresponding finite reaction sequence $\llbracket\left\langle\mathcal{B}\right.$, nil $\left.; P_{2}\right\rangle \rrbracket \rightarrow+\llbracket\left\langle\mathcal{B}^{\prime}, P_{2}^{\prime}\right\rangle \rrbracket$.

Proof. Assume $\left\langle\mathcal{B}, P_{2}\right\rangle \rightarrow$. Proof by structural induction on $\llbracket \mathcal{B}, P_{2} \rrbracket \rightarrow \rightarrow^{+} \llbracket\left\langle\mathcal{B}^{\prime}, P_{2}^{\prime}\right\rangle \rrbracket$.

Similar to other cases, if we cannot reduce a sequence the failure is propagated up-the-tree through the reaction rule seq_fail (Fig. 14c). Importantly, the reaction rule seqfail - and later failure cases - do not require the lefthand entity to be under a Reduce. This means the reaction can be applied as soon as a failure is discovered rather than the next time the agent attempts to advance the intention. This matches the CAN semantics that handle the failure of intention immediately ( $\Delta_{\perp}$ in Fig. 6- encoded in the next section).

When using the reactions together we need to carefully manage their ordering. The reaction rule reduce_seq is a generalisation of seq_succ and seq_fail. For example, if we get rid of id under the control Seq (not id under Cons) on the left-hand side of reaction rule reduce_seq, we get the reaction rule seq_succ. Therefore, we enforce a priority ordering on the reaction rules as given in Fig. 14 to ensure that the special case are applied when they need to.

\subsubsection{Failure Recovery}

We now encode the derivation rules $\nabla_{;}, \nabla_{\top}$, and $\nabla_{\perp}$ that relate to failure recovery

The reaction rule try_seq (Fig. 15a) encodes the derivation rule $\triangleright$; by pushing reduction into the left of the $\triangleright$ operator if no failure occurs. 
Lemma 6. (Faithfulness of $\triangleright_{;}$) $\triangleright_{\text {; }}$ has a corresponding finite reaction sequence $\llbracket\left\langle\mathcal{B}, P_{1} \triangleright P_{2}\right\rangle \rrbracket \rightarrow^{+} \llbracket\left\langle\mathcal{B}^{\prime}, P_{1}^{\prime} \triangleright P_{2}\right\rangle \rrbracket$.

Proof. Structural induction on $\llbracket \mathcal{B}, P_{1} \rrbracket \rightarrow^{+} \llbracket\left\langle\mathcal{B}^{\prime}, P_{1}^{\prime}\right\rangle \rrbracket$.

If the selected plan was executed successfully, the reaction rule try_succ (in Fig. 15b encodes the derivation rule $\triangleright_{\top}$ to propagate success up-the-tree by removing the $\triangleright$ structure.

Lemma 7. (Faithfulness of $\triangleright_{\mathrm{T}}$ ) $\left\langle\mathcal{B}\right.$, nil $\left.\left.\triangleright P_{2}\right\rangle\right\rangle \stackrel{\triangleright_{\mathrm{T}}}{\longrightarrow}\left\langle\mathcal{B}^{\prime}\right.$, nil $\left.\triangleright P_{2}\right\rangle$, has a corresponding finite reaction sequence $\llbracket\left\langle\mathcal{B}\right.$, nil $\left.\triangleright P_{2}\right\rangle \rrbracket \rightarrow^{+} \llbracket\left\langle\mathcal{B}^{\prime}\right.$, nil $\rangle \rrbracket$.

Proof. $\rightarrow^{+}$corresponds to $\stackrel{\text { try_suc }}{\longrightarrow}$. Trivial.

Finally, the reaction rule try_failure (depcited in Fig. 15c) encodes the derivation rule $\nabla_{\perp}$. This is the first instance where ReduceF is used as a premise to denote a program which failed to progress. To recover, the failed program is deleted and the agent tries to reduce the right-hand side of $\triangleright$ (i.e. by choosing from the remaining the set of relevant plans).

Lemma 8. (Faithfulness of $\triangleright_{\perp}$ ) $\triangleright_{\perp}$ has a corresponding finite reaction sequence $\llbracket\left\langle\mathcal{B}, P_{1} \triangleright P_{2}\right\rangle \rrbracket \rightarrow \rightarrow^{+} \llbracket\left\langle\mathcal{B}^{\prime}, P_{2}^{\prime}\right\rangle \rrbracket$.

Proof. Assume $\left\langle\mathcal{B}, P_{1}\right\rangle \nrightarrow$, and $\left\langle\mathcal{B}, P_{2}\right\rangle \rightarrow\left\langle\mathcal{B}, P_{2}^{\prime}\right\rangle .\left\langle\mathcal{B}, P_{1}\right\rangle \nrightarrow$ implies that ReduceF holds as the premise required by the match. Structural induction on $\llbracket\left\langle\mathcal{B}, P_{2}\right\rangle \rrbracket \rightarrow \rightarrow^{+} \llbracket\left\langle\mathcal{B}^{\prime}, P_{2}^{\prime}\right\rangle \rrbracket$ completes the proof.

If no backup plans apply e.g. when there are no plans left to select, the failure is pushed upwards through the reaction rule select_plan_F (Fig. 13c). The reaction rule try_failure does not apply as the Cons entity has been removed by the point. Finally, as with sequencing, an priority order is required as try_seq generalises the other reactions.

\subsubsection{Agent Steps}

To complete the core semantics of CAN, we now encode the agent-level derivation rules: $A_{\text {event }}, A_{\text {step }}$, and $A_{\text {update }}$.

The derivation rule $A_{\text {event }}$ allows the agent to respond to an external event by adopting it in the intention base. It is encoded as reaction rule A_event (depicted in Fig. 16a) that simply moves the event from being a desire to being an intention.

Lemma 9. (Faithfulness of $A_{\text {event }}$ ) $A_{\text {event }}$ has a corresponding finite reaction sequence $\llbracket\left\langle E^{e}, \mathcal{B}, \Gamma\right\rangle \rrbracket \rightarrow \rightarrow^{+} \llbracket\left\langle E^{e} \backslash e_{n}, \mathcal{B}^{\prime}, \Gamma \cup e_{n}\right\rangle \rrbracket$.

Proof. $\rightarrow{ }^{+}$corresponds to $\stackrel{\text { A_event }}{\longrightarrow}$. Trivial.

The derivation rule $A_{\text {step }}$ allows the agent to execute a given intention one step further, i.e. one reduction step. To encode it, we have reaction rule intention_step (in Fig. 16b that pushes a reduction into an intention 


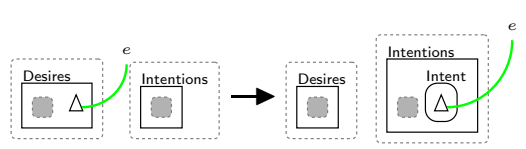

(a) A_event

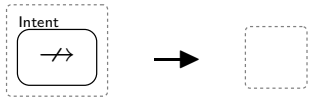

(c) intention_done_F

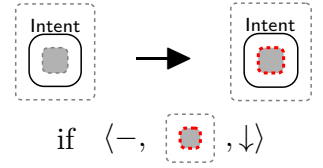

(b) intention_step

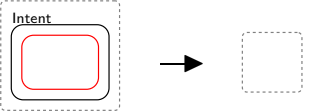

(d) intention_done_succ

Figure 16: Agent level reactions with priorities: $\{$ A_event, intention_step $<<$ \{intention_done_F, intention_done_succ $\}$

(down-the-tree) if it is not already being reduced. This rule introduces the reduction form $\llbracket \mathbb{l}$ to an intention.

If the reduction below is successful we are left with a new updated $P^{\prime}$ (and $\left.\mathcal{B}^{\prime}\right)$ as required. Unlike the CAN derivation rule which removes the old intention and replaces it with a modified intention, ours is updated in-place. Also multiple intentions can be reduced concurrently, e.g. intention_step can be applied to two different intentions. However, any single intention can only have one Reduce entity (as per the condition on the rule).

Lemma 10. (Faithfulness of $A_{\text {step }}$ ) $A_{\text {step }}$ has a corresponding finite reaction sequence $\llbracket\left\langle E^{e}, \mathcal{B}, \Gamma\right\rangle \rrbracket \rightarrow \rightarrow^{+} \llbracket\left\langle E^{e}, \mathcal{B}^{\prime}, \Gamma \backslash P \cup P^{\prime}\right\rangle \rrbracket$.

Proof. Structural induction on $\llbracket \mathcal{B}, P \rrbracket \rightarrow \rightarrow^{+} \llbracket\left\langle\mathcal{B}^{\prime}, P^{\prime}\right\rangle \rrbracket$. To enable the finite reaction sequence $\llbracket \mathcal{B}, P \rrbracket \rightarrow+\llbracket\left\langle\left\langle\mathcal{B}^{\prime}, P^{\prime}\right\rangle \rrbracket\right.$, a reduction entity Reduce is introduced. That is we move between $\Rightarrow$ and $\rightarrow$ rules.

The derivation rule $A_{\text {update }}$ is encoded by reaction rules intention_done_F (Fig. 16c) and intention_done_succ (Fig. 16d). The reaction rule intention_done_F handles the case there was a failure to progress an intention. That is, if after pushing a reduction into the intention (via intention_step), we eventually receive Intent.ReduceF. The reaction rule intention_done_succ is a special case of intention_done_F for the situation a intention completed successfully (returned Intent.1). As the $A_{\text {update }}$ rule only applies on failure to reduce an intention, intention_done_succ matches the form where we have tried to reduce an intention with the nil program inside. Importantly, this means that if an intention finishes an execution with $P=n i l$, it is not until the next attempt to reduce it that $A_{\text {update }}$ is applied. This mirrors the CAN semantics that cannot tell if an intention is removed because it is finished, or if it failed.

Lemma 11. (Faithfulness of $\left.A_{\text {update }}\right) A_{\text {update }}$ has a corresponding finite reaction sequence $\llbracket\left\langle E^{e}, \mathcal{B}, \Gamma\right\rangle \rrbracket \rightarrow+\llbracket\left\langle E^{e}, \mathcal{B}^{\prime}, \Gamma \backslash P\right\rangle \rrbracket$.

Proof. The proof is given in two cases:

Case 1: If $\langle\mathcal{B}, P\rangle \nrightarrow$, failure to reduce implies that Intent. ReduceF is matched. Therefore, $\rightarrow+$ corresponds to $\stackrel{\text { intention_done_F }}{\longrightarrow}$. 


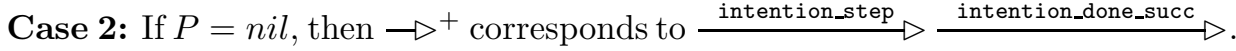
No other rule applies as there is no way to reduce Reduce.1 further.

To ensure agent-level transition only apply if we are not currently reducing an intention both intention_step and A_event are in the lowest priority class. For the same reasons as other failure recovery rules, intention_done_F and intention_done_succ are in a higher priority class so ensure they fire at the end of any failed steps.

\subsection{Proof of Correctness}

In the previous sections, we established that the CAN derivation rules can be encoded by a finite sequence of reaction rules that do not introduce new branching (i.e. CAN derivation rules) which is not present in the starting state. We now prove the following.

Theorem 1 (Faithfulness). For each CAN step $\left\langle E^{e}, \mathcal{B}, \Gamma\right\rangle \Rightarrow\left\langle E^{\prime e}, \mathcal{B}^{\prime}, \Gamma^{\prime}\right\rangle$ there exists a finite sequence of reactions such that $\llbracket\left\langle E^{e}, \mathcal{B}, \Gamma\right\rangle \rrbracket \rightarrow+\llbracket\left\langle E^{\prime e}, \mathcal{B}^{\prime}, \Gamma^{\prime}\right\rangle \rrbracket$.

Proof. The proof is the collection of the proofs of Lemma 9 - 11: all three possible agent steps have corresponding finite reaction sequences and do not introduce new branching. Therefore, regardless of the step the agent takes, it is guaranteed to have a faithful encoding.

\subsection{Reduction Example}

To show how reduction works, in particular how failures are propagated through the AND/OR tree, we re-visit our running conference travelling example to address external event $e_{1}$. Consider the following configuration:

$$
\text { Agent }=\left\langle\mathcal{B}=\left\{b_{1}, b_{2}, b_{6}, b_{7}\right\}, P=e_{1}\right\rangle
$$

This configuration has the current belief base $\mathcal{B}$ and current intention $P=e_{1}$. The bigraph (omitting desires and plan library) is:

$$
\llbracket \text { Agent } \rrbracket=\text { Beliefs. }(\mathrm{B}(1)|\mathrm{B}(2)| \mathrm{B}(6) \mid \mathrm{B}(7)) \| \text { Intent. } \mathrm{E}_{e_{1}}
$$

The detailed reduction step is given in Fig. 17. For succinctness, whenever appropriate, we use the mapping function to denote the part of bigraphical encoding e.g. $\llbracket P l_{2} \rrbracket$ while keeping the belief base implicitly as the background. The top-side of the reaction rule indicates the reaction rule that is applied and the bottom-side of the reaction rule indicates the result of application of the reaction rule, with line number () in the beginning of each line. A short commentary is as follows. In (1), the agent starts with an event to address. The reaction rule intention_step introduces the entity Reduce. (2) and (3) show that to reduce an event, the event is replaced with its relevant plans. Reaction rule intention_step once again introduces Reduce for selection of an applicable plan. (4) to (6) shows the successful selection of an applicable plan, plan $P l_{1}$. From (8) to (9), the reaction rule try_seq pushes reduction in the left-hand side of the $\triangleright$ symbol, and from $(9)$ to $(10)$, the reaction rule reduce_seq pushes the 
reduction into the first child of a sequence. (10) to (12) shows the execution of an action. In this case, we can see that the pre-condition of the action is not met, thus producing the entity ReduceF. As a consequence, this triggers failure recovery by deleting the failed program Finally, (13) to (16) provides the successful re-selection of another applicable plan, namely plan $\mathrm{Pl}_{2}$.

\section{Extended Features}

The full CAN language also supports concurrency within plan-bodies, and declarative goals which allow an event to be repeatedly pursued until specified success/failure conditions holds. We now show how these features are encoded as bigraph reaction rules.

\subsubsection{Concurrency}

The CAN semantics for concurrency are given in Fig. 18. They allow two branches within a single AND/OR tree to be reduced concurrently. For example, concurrency allows an agent to pursue two sub-tasks (i.e. two sub-events) but the ordering does not matter as long as they are all achieved eventually. However, the actual reduction is not in parallel. Instead the agent chooses to either reduce the left or right branch on each step (i.e. they are interleaved). This matches common agent implementations that do not support multi-core processing.

Two reaction rules conc_L (Fig. 19a), and conc_R (Fig. 19b) encode concurrency. As they have the same priority, these rules specify that reduction can be pushed down either the left or right branch.

Lemma 12. (Faithfulness of $\|_{1}$ and $\|_{2}$ ) $\|_{1}$ and $\|_{2}$ have a corresponding finite reaction sequence $\llbracket\left\langle\mathcal{B}, P_{1} \| P_{2}\right\rangle \rrbracket \rightarrow \rightarrow^{+} \llbracket\left\langle\mathcal{B}^{\prime}, P_{1}^{\prime} \| P_{2}\right\rangle \rrbracket$ and $\llbracket\left\langle\mathcal{B}, P_{1} \| P_{2}\right\rangle \rrbracket \rightarrow \rightarrow^{+} \llbracket\left\langle\mathcal{B}^{\prime}, P_{1} \|\right.$ $\left.P_{2}^{\prime}\right\rangle \rrbracket$, respectively.

Proof. The proof of $\|_{1}$ is given in the following two cases:

Case 1. If $P_{2}=n i l$ then $\left\langle\mathcal{B}, P_{2}\right\rangle \nrightarrow$. The reaction rule conc_nil_L in Fig. 19c applies. The proof completes by induction on $\llbracket \mathcal{B}, P_{1} \rrbracket$.

Case 2. If $P_{1} \neq$ nil then the reaction rule conc $\_$applies. The proof completes by induction on $\llbracket \mathcal{B}, P_{1} \rrbracket$.

The proof of $\|_{2}$ can be given in a similar way.

Concurrent programs are considered successfully completed when both branches complete, i.e. reduced to $\mathrm{nil}$. The reaction rule conc_suc is given in Fig. 19e to handles the completion of concurrent programs.

Lemma 13. (Faithfulness of $\|_{T}$ ) $\|_{T}$ has a corresponding finite reaction sequence $\llbracket\langle\mathcal{B}$, nil $\|$ nil $\rangle \rrbracket \rightarrow{ }^{+} \llbracket\left\langle\mathcal{B}^{\prime}\right.$, nil $\rangle \rrbracket$.

Proof. $\longrightarrow+$ corresponds to $\stackrel{\text { conc_suc }}{\longrightarrow}$. Trivial.

As always for the case of failures, additional reaction rules conc_fail_L (Fig. 19f) and conc_fail_R (Fig. 19g) propagate failure up-the-tree if either of the two 
(1) Intent. $E_{e_{1}}$ intention_step

(2) Intent.Reduce. $E_{e_{1}}$ reduce_event

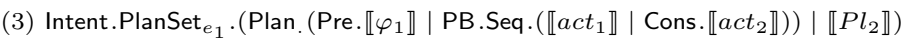
$\stackrel{\text { intention_step }}{\longrightarrow}$

(4) Intent.Reduce.PlanSet $e_{1} \cdot\left(\right.$ Plan. (Pre. $\llbracket \varphi_{1} \rrbracket \mid$ PB.Seq. $\left(\llbracket a c t_{1} \rrbracket \mid\right.$ Cons. $\left.\left.\left.\llbracket a c t_{2} \rrbracket\right)\right) \mid \llbracket P l_{2} \rrbracket\right)$ select_plan_check

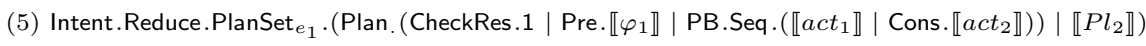
set_ops *

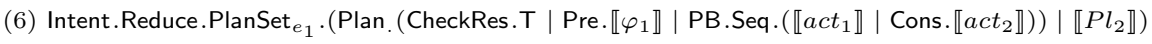
select_plan_T

(7) Intent.Try.(Seq. $\left(\llbracket a c t_{1} \rrbracket \mid\right.$ Cons. \act $\left.2 \rrbracket\right) \mid$ Cons.PlanSet $\left._{e_{1}} \cdot \llbracket P l_{2} \rrbracket\right)$ $\stackrel{\text { intention_step }}{\longrightarrow}$

(8) Intent.Reduce.Try.(Seq. $\left(\llbracket a c t_{1} \rrbracket \mid\right.$ Cons. $\left.\llbracket a c t_{2} \rrbracket\right) \mid$ Cons.PlanSet $\left._{e_{1}} \cdot \llbracket P l_{2} \rrbracket\right)$ $\stackrel{\text { try_seq }}{\longrightarrow}$

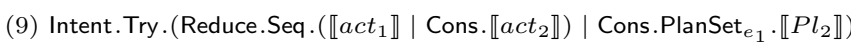
reduce_seq

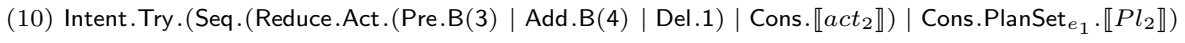
act_check

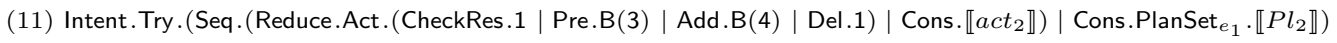
$\stackrel{\text { set_ops }}{\longrightarrow}{ }^{*}$

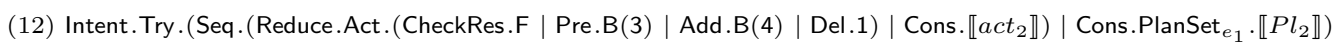
$\stackrel{\text { act_F }}{\longrightarrow}\left(\right.$ as $\left.\mathcal{B} \not \models b_{3}\right)$

(13) Intent.Try.(ReduceF | Cons.PlanSet $\left.\left.e_{1} \cdot \llbracket P l_{2} \rrbracket\right)\right)$ try_failure

(14) Intent.Reduce.PlanSet $e_{1} \cdot \llbracket P l_{2} \rrbracket$ select_plan_check

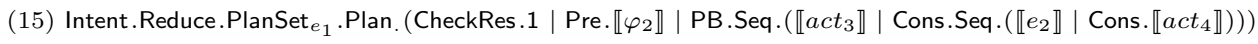
select_plan_T

(16) Intent.Try.(Seq. $\left(\llbracket a c t_{3} \rrbracket \mid\right.$ Cons.Seq. $\left(\llbracket e_{2} \rrbracket \mid\right.$ Cons. $\left.\left.\llbracket a c t_{4} \rrbracket\right)\right) \mid$ Cons.PlanSet $\left._{e_{1}} .1\right)$

Figure 17: Example bigraphical reduction of event $e_{1}$ from Fig. 2 


$$
\begin{gathered}
\frac{\left\langle\mathcal{B}, P_{1}\right\rangle \rightarrow\left\langle\mathcal{B}^{\prime}, P_{1}^{\prime}\right\rangle}{\left\langle\mathcal{B},\left(P_{1} \| P_{2}\right)\right\rangle \rightarrow\left\langle\mathcal{B}^{\prime},\left(P_{1}^{\prime} \| P_{2}\right)\right\rangle} \|_{1} \\
\frac{\left\langle\mathcal{B}, P_{2}\right\rangle \rightarrow\left\langle\mathcal{B}^{\prime}, P_{2}^{\prime}\right\rangle}{\left\langle\mathcal{B},\left(P_{1} \| P_{2}\right)\right\rangle \rightarrow\left\langle\mathcal{B}^{\prime},\left(P_{1} \| P_{2}^{\prime}\right)\right\rangle} \|_{2} \\
\frac{\langle\mathcal{B},(\text { nill } \| \text { nil })\rangle \rightarrow\langle\mathcal{B}, \text { nil }\rangle}{\top} \|_{\top}
\end{gathered}
$$

Figure 18: CAN concurrency rules.

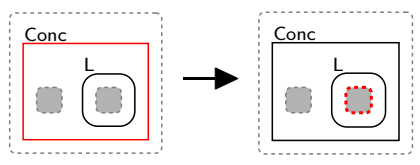

(a) conc_L

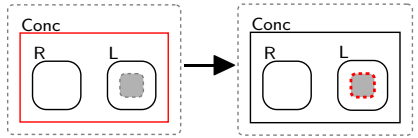

(c) conc_nil_L

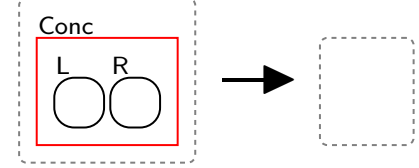

(e) conc_suc

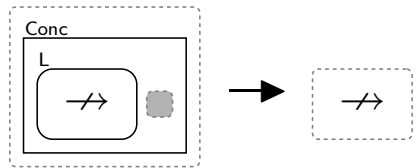

(f) conc_fail_L

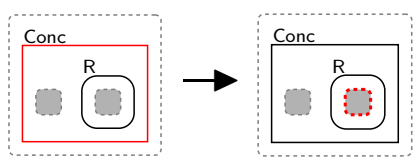

(b) conc $R$

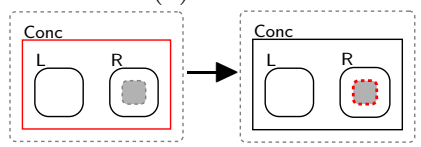

(d) conc_nil_R

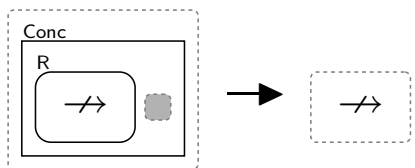

(g) conc_fail_R

Figure 19: Reactions for concurrency with priorities: $\quad\left\{\right.$ conc_L, conc_R $_{2}<$ $\{$ conc_nil_L, conc_nil_R $\}<\{$ conc_suc, conc_fail_L, con_fail_R $\}$ 


$$
\begin{aligned}
& \frac{\mathcal{B} \models \varphi_{s}}{\left\langle\mathcal{B}, \operatorname{goal}\left(\varphi_{s}, P, \varphi_{f}\right)\right\rangle \rightarrow\langle\mathcal{B}, \text { nil }\rangle} G_{s} \quad \frac{\mathcal{B} \models \varphi_{f}}{\left\langle\mathcal{B}, \operatorname{goal}\left(\varphi_{s}, P, \varphi_{f}\right)\right\rangle \rightarrow\langle\mathcal{B}, \text { ?false }\rangle} G_{f} \\
& \frac{P \neq P_{1} \triangleright P_{2} \quad \mathcal{B} \not \models \varphi_{s} \quad \mathcal{B} \not \models \varphi_{f}}{\left\langle\mathcal{B}, \operatorname{goal}\left(\varphi_{s}, P, \varphi_{f}\right)\right\rangle \rightarrow\left\langle\mathcal{B}, \operatorname{goal}\left(\varphi_{s}, P \triangleright P, \varphi_{f}\right)\right\rangle} G_{\text {init }} \\
& \frac{\mathcal{B} \not \models \varphi_{s} \quad \mathcal{B} \not \models \varphi_{f}}{\left\langle\mathcal{B}, P_{1}\right\rangle \rightarrow\left\langle\mathcal{B}^{\prime}, \mathcal{A}^{\prime}, P_{1}^{\prime}\right\rangle} \overline{\left\langle\mathcal{B}, \operatorname{goal}\left(\varphi_{s}, P_{1} \triangleright P_{2}, \varphi_{f}\right)\right\rangle \rightarrow\left\langle\mathcal{B}^{\prime}, \mathcal{A}^{\prime}, \operatorname{goal}\left(\varphi_{s}, P_{1}^{\prime} \triangleright P_{2}, \varphi_{f}\right)\right\rangle} G ; \\
& \frac{\mathcal{B} \not \models \varphi_{s} \quad \mathcal{B} \not \models \varphi_{f} \quad\left\langle\mathcal{B}, P_{1}\right\rangle \nrightarrow}{\left\langle\mathcal{B}, \operatorname{goal}\left(\varphi_{s}, P_{1} \triangleright P_{2}, \varphi_{f}\right)\right\rangle \rightarrow\left\langle\mathcal{B}, \operatorname{goal}\left(\varphi_{s}, P_{2} \triangleright P_{2}, \varphi_{f}\right)\right\rangle} G_{\triangleright}
\end{aligned}
$$

Figure 20: Derivation rules for declarative goals.

concurrent branches results in a failure. Importantly, we fail as soon as either branch fails rather than waiting for the second branch to complete (either successfully or with failure). That is, we short-circuit computation as required.

As before, the priority ordering on the reaction rules is required as some reaction rules generalise others, e.g. the reaction rule conc $R$ would also match reaction rule conc_succ.

\subsubsection{Declarative Goals}

Declarative goals allow an agent to persistently respond to some event $e$ until either the success or failure conditions are met. The CAN semantics for declarative goals are given in Fig. 20 The derivation rules $G_{s}$ and $G_{f}$ deal with the cases when either the success condition $\varphi_{s}$ or the failure condition $\varphi_{f}$ become true. The derivation rule $G_{\text {init }}$ initialises persistence by setting the program in the declarative goal to be $P \triangleright P$, i.e. if $P$ fails try $P$ again. The derivation rule $G$; takes care of performing a single step on an already initialised program. Finally, the derivation rule $G_{\triangleright}$ re-starts the original program if the current program has finished or got blocked (when neither $\varphi_{s}$ nor $\varphi_{f}$ becomes true).

To reduce the number of reaction rules for encoding declarative goals, we check both success and failure conditions simultaneously through reaction rule goal_check (Fig. 21a). As before, the entailment machinery provides atomic checks in both cases. Afterwards the reaction rules goal_suc (Fig. 21b) and goal_fail (Fig. 21c) determine if the goal should complete (either successfully or with failure). Strictly speaking it is possible both success/failure conditions hold simultaneously, however in practice it is usually assumed success/failure conditions are mutually exclusive.

An interesting feature of the CAN derivation rule $G_{f}$ is the use of ?false in the resulting state. This plays a similar role to ReduceF by explicitly creating an irreducible term to trigger further handling up-the-tree. Recall that the belief entailment in derivation rule ? can be regarded as the special case of the derivation rule act (Section 3). Therefore, we simply let goal_fail reduce to an Act with a false precondition (that always fails) to indicate a failure. 


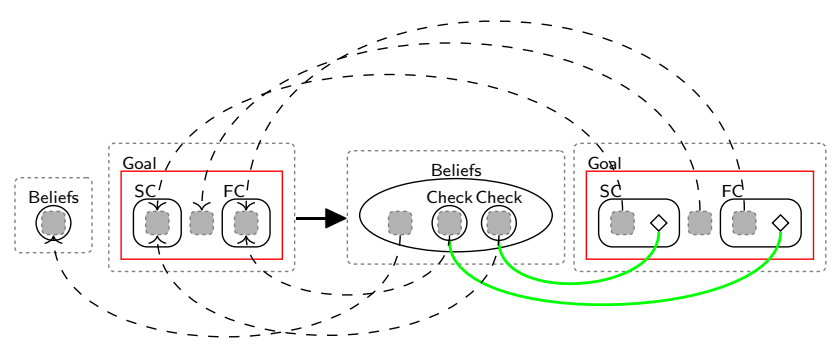

(a) goal_check

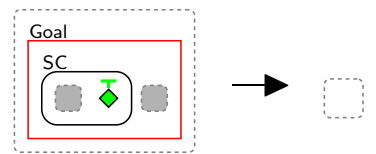

(b) goal_suc

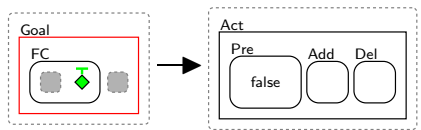

(c) goal_fail

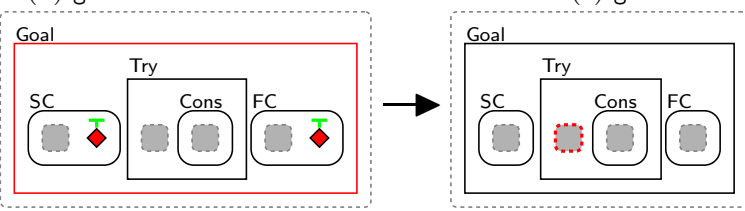

(d) goal_reduce

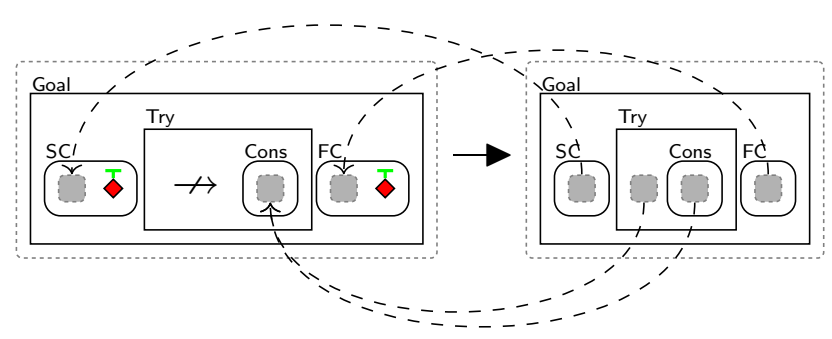

(e) goal_persist

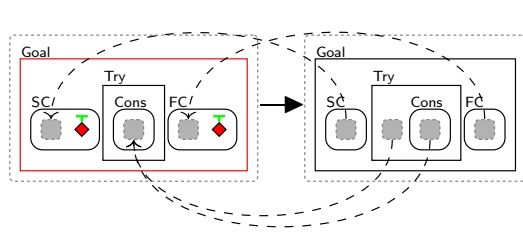

(f) goal_persist_nil

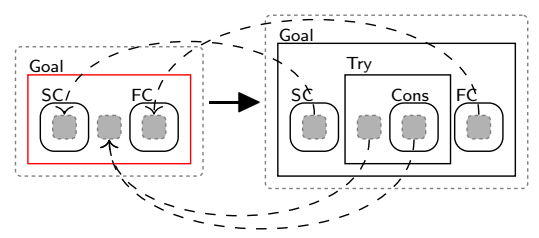

(g) goal_init

Figure 21: Reactions for declarative goals with priorities: goal_init < \{goal_reduce, goal_check, goal_fail, goal_suc $\}<$ goal_persist, goal_persist_nil $\}$. 
Lemma 14. (Faithfulness of $G_{s}$ ) $G_{s}$ has a corresponding finite reaction sequence $\llbracket\left\langle\mathcal{B}, \operatorname{goal}\left(\varphi_{s}, P, \varphi_{f}\right)\right\rangle \rrbracket \rightarrow \rightarrow^{+} \llbracket\langle\mathcal{B}$, nil $\rangle \rrbracket$.

Proof. $\longrightarrow \longrightarrow^{+}$corresponds to $\stackrel{\text { goal_check }}{\longrightarrow} \stackrel{\{\text { set_ops }\}}{\longrightarrow} \triangleright^{*} \stackrel{\text { goal_suc }}{\longrightarrow}$. As the checks are finite, atomic, and over auxiliary bigraphical entities the number of reactions is finite and does not introduce additional branching as required.

Lemma 15. (Faithfulness of $G_{f}$ ) $G_{f}$ has a corresponding finite reaction sequence $\llbracket\left\langle\mathcal{B}, \operatorname{goal}\left(\varphi_{s}, P, \varphi_{f}\right)\right\rangle \rrbracket \rightarrow+\llbracket\langle\mathcal{B}, ?$ false $\rangle \rrbracket$.

Proof. $\rightarrow+$ corresponds to $\stackrel{\text { goal_check }}{\longrightarrow} \stackrel{\text { \{set_ops }\}}{\longrightarrow}{ }^{*} \stackrel{\text { goal_fail }}{\longrightarrow}$. As the checks are finite, atomic, and over auxiliary bigraphical entities the number of reactions is finite and does not introduce additional branching as required.

Similar to the derivation rule $\triangleright$; , the derivation rule $G$; reduces the leftbranch of the symbol $\triangleright$. The reaction goal_reduce (Fig. 21d) pushes the reduction down the left-branch. To ensure the ordering between rules $G_{s}, G_{f}$, and $G_{\text {; }}$, we explicitly match only on the case that the checks have already been performed. In other words, the goal will only be pursued if neither the success or failure condition holds.

Lemma 16. (Faithfulness of $\left.G_{;}\right) G_{;}$, has a corresponding finite reaction sequence $\llbracket\left\langle\mathcal{B}, \operatorname{goal}\left(\varphi_{s}, P_{1} \triangleright P_{2}, \varphi_{f}\right)\right\rangle \rrbracket \rightarrow^{+} \llbracket\left\langle\mathcal{B}^{\prime}, P_{1}^{\prime} \triangleright P_{2}\right\rangle \rrbracket$.

Proof. Finite reduction on $\llbracket \mathcal{B}, P_{1} \rrbracket \rightarrow^{+} \llbracket\left\langle\mathcal{B}^{\prime}, P_{1}^{\prime}\right\rangle \rrbracket$.

The derivation rule $G_{\triangleright}$ likewise is very similar to the derivation rule $\triangleright_{\perp}$. Unlike in rule $\triangleright_{\perp}$, however, in rule $G_{\triangleright}$ we keep the $\triangleright$ structure in-place and replicate $P_{2}$, thus giving the declarative goals their persistence. The can reaction rule goal_persist (Fig. 21e $)$ encodes this case with the match of ReduceF ensuring the premise $\left\langle\mathcal{B}, P_{1}\right\rangle \nrightarrow$ holds. Through duplication, we decouple the failure of the plan execution from the failure of the goal (as specified by success/failure conditions). Finally, an addition reaction goal_persist_nil (Fig. 21f) enables the agent to persists even in the the case where the program executed successfully (but the goal success/failure did not hold).

Lemma 17. (Faithfulness of $G_{\triangleright}$ ) $G_{\triangleright}$ has a corresponding finite reaction sequence $\llbracket\left\langle\mathcal{B}, \operatorname{goal}\left(\varphi_{s}, P_{1} \triangleright P_{2}, \varphi_{f}\right)\right\rangle \rrbracket \rightarrow \rightarrow^{+} \llbracket\left\langle\mathcal{B}, P_{2} \triangleright P_{2}\right\rangle \rrbracket$.

Proof. $\rightarrow+$ corresponds to $\stackrel{\text { goal_persist }}{\longrightarrow}$ or $\stackrel{\text { goal_persist_nil }}{\longrightarrow}$. Rules are mutually exclusive.

The derivation rule $G_{i n i t}$ is encoded through reaction goal_init that sets up the required $\triangleright$ structure. To ensure this is applied at the right time, we have priority classes with goal_init $<$ \{goal_persist, goal_reduce $\}$ to ensure the premise $P \neq P_{1} \triangleright P_{2}$ holds.

Lemma 18. (Faithfulness of $G_{\text {init }}$ ) $G_{\text {init }}$ has a corresponding finite reaction sequence $\llbracket\left\langle\mathcal{B}, \operatorname{goal}\left(\varphi_{s}, P, \varphi_{f}\right)\right\rangle \rrbracket \rightarrow+\llbracket\langle\mathcal{B}, P \triangleright P\rangle \rrbracket$. 
Proof. $\rightarrow+$ corresponds to $\stackrel{\text { goal_init }}{\longrightarrow}$. Priority classes of reactions ensure $P \neq P_{1} \triangleright P_{2}$ as required.

For declarative goals, due to persistence, there is no additional rule that propagates failures upwards7.

As with the previous lemmas these, extended features can be integrated easily into Theorem 1 to prove the extended semantics is also faithful. As the theorem is over agent-level transitions the theorem itself does not change, but the set of possible finite $\rightarrow+$ sequences increases.

\section{Examples: UAVs}

To illustrate our modelling and verification framework, we consider three examples taken from UAV surveillance and retrieval mission systems. The examples cover persistent patrol, concurrent sensing, and contingency handling in object retrieval and highlight the three distinguishing features of CAN: declarative goals, concurrency, and failure recovery.

\subsection{Persistent Patrol}

UAVs are used in surveillance operations, with a UAV patrolling a predefined area to identify objects of interest. The UAV can request refuelling when the battery is low, and parking mode should be activated when there is harsh weather.

The agent design and its corresponding bigraphical encoding is in Fig. 22 The external event e_init1 (line 4) initiates persistent patrol. There is only one plan (line 6) relevant to e_init1, whose context is always true (represented by an empty region bigraph 1 ), thus always applicable, and whose plan-body is declarative goal goal (false,e_patrol_task,false). The event e_patrol_task is persistent because the success and failure conditions never hold, that is, we have an infinite process executing e_patrol_task. In practice, we require some flexibility in case of low battery or harsh weather. The plan for e_patrol_task (line 7 ) indicates the patrol task may need to be paused (i.e. followed by the event e_pause), when the success condition is true, i.e. when battery_low or harsh_weather holds (added to the belief base). If the pause is required and after achieving event e_pause (lines 9-10), the event e_patrol_task will be pursued again. For succinct presentation, we note that the encoding of action such as patrol and wait are not shown, but can be found in our model [27].

\subsection{Concurrent Sensing}

UAVs may also be used for sensing tasks. In this case we consider a UAV that analyses dust particles, and performs aerial photo collection, e.g. for analysis in post volcanic eruptions.

\footnotetext{
${ }^{7}$ Meeting the failure conditions does eventually lead to failure but requires additional steps.
} 


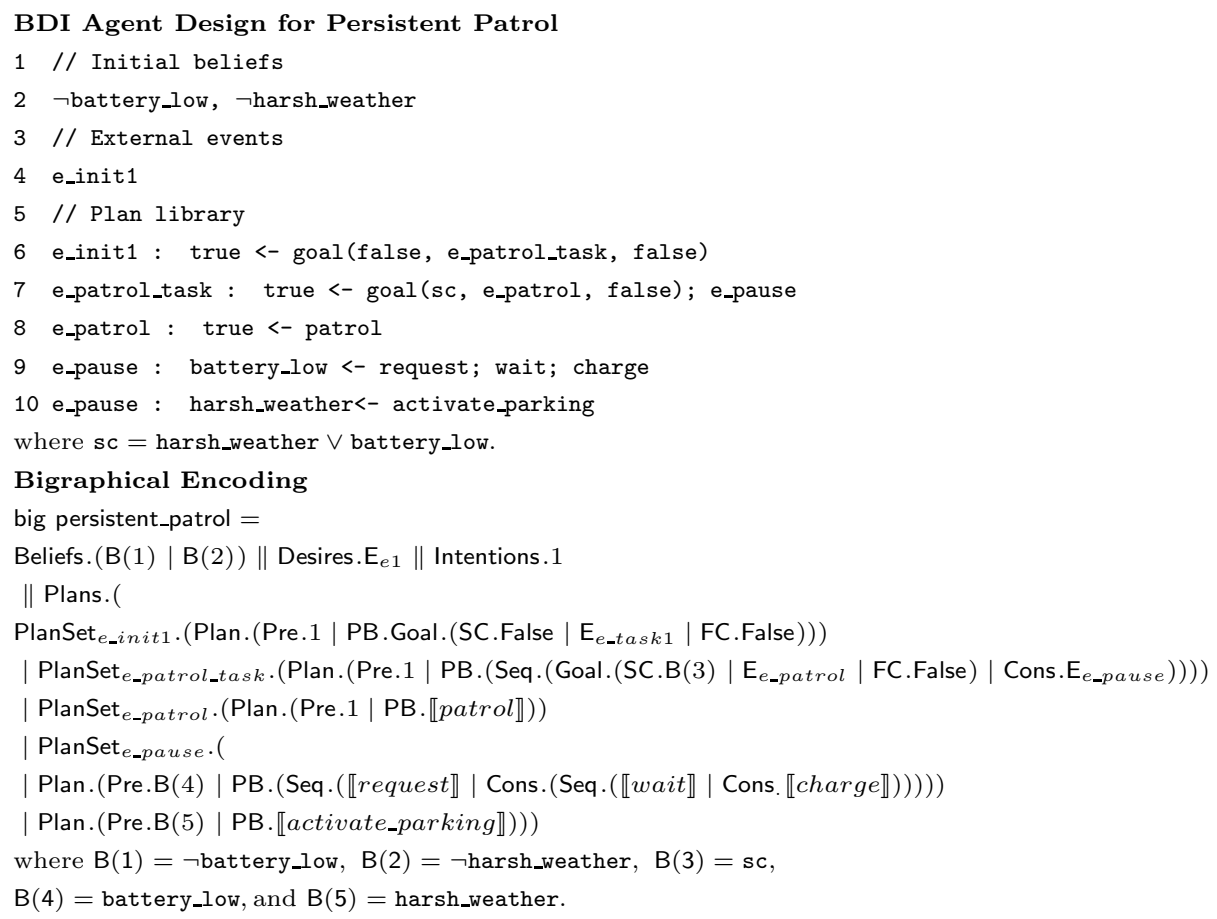

Figure 22: Persistent Patrol: BDI Agent Design and Bigraphical Encoding.

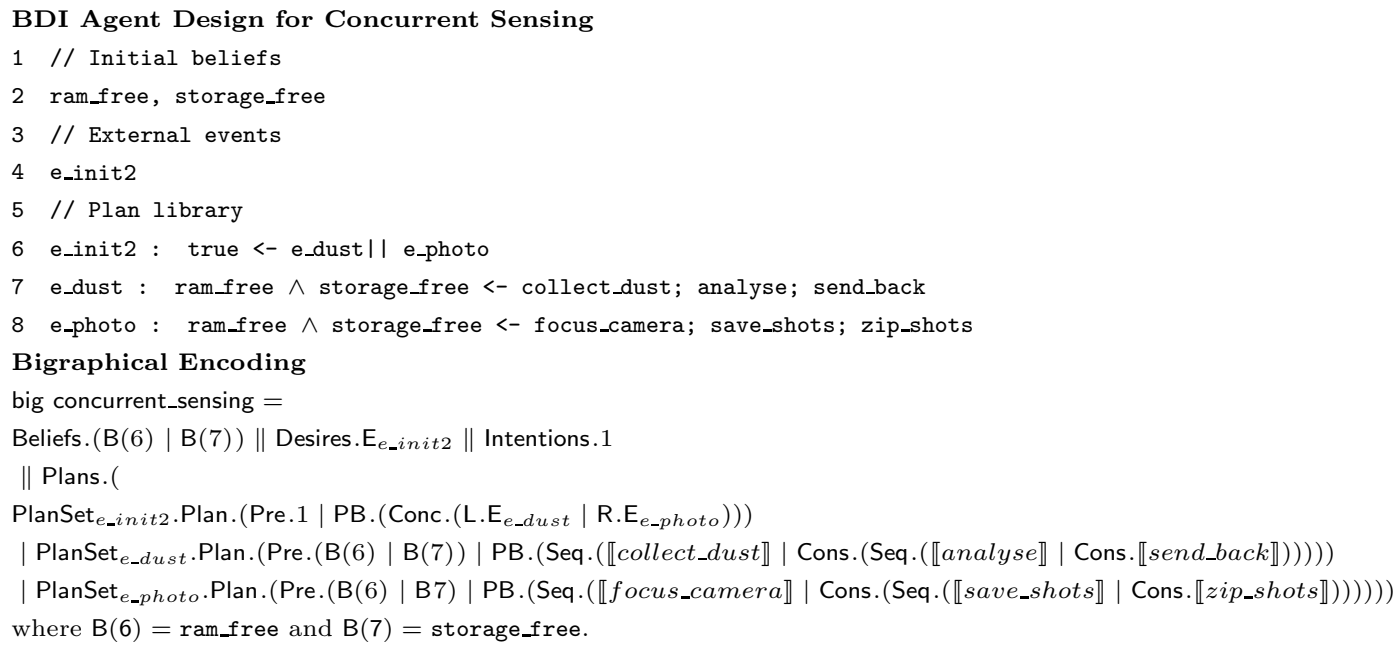

Figure 23: Concurrent Sensing: BDI Agent Design and Bigraphical Encoding. 
An agent design to achieve this concurrent sensing task is in Fig. 23. The external event e_init2 (line 4) initiates the mission and the relevant plan (line 6) has tasks for dust monitoring (e_dust) and photo collection (e_photo) as the concurrent programs in the plan-body. The on-board dust sensors require high-speed RAM to collect and analyse the data, hence condition ram_free, and when the analysis is complete, results are written to storage (hence condition storage_free), and sent back to the control. Similarly, to collect aerial photos, the UAV reserves and focuses the camera array (focus_camera), then camera shots are compressed (zip_shot), and sent back. Recall, for successful completion, both concurrent tasks have to complete successfully.

\subsection{Contingency Handling for a Retrieve Task}

UAVs may be used for object retrieval tasks, e.g. package delivery. An agent design for retrieval is in Fig. 24. It has one (retrieve) task, initiated by external event e_retrv (line 4), which may be affected by engine or sensor malfunction, Event e_retrv is handled by five relevant plans available (lines 6 to 10). The first 3 plans provide different flight paths after take-off, in which case the failure condition is (subsequent) engine or sensor malfunction. The last 2 plans (line 9 and 10) indicate safe recovery in the event of engine or sensor malfunction.

\section{Properties}

To verify the designs, we generate a transition system from the BRS representing the agents (and their semantics). The transistion system has bigraphs as states and reactions as transitions. We can reason about static properties using bigraph patterns [20] and dynamic properties using linear or branching time temporal logics such as Computation Tree logic (CTL) [28], which we use in our examples.

\subsection{Bigraph patterns}

Bigraph patterns are predicates on states: if the pattern matches the current state then the predicate is true.

We have found the bigraph patterns most useful for reasoning about BDI agents are often a fragment of the right-hand side of reactions, i.e. they check that a desired or anticipated operation has taken place. For example, consider the state predicate: there is a declarative goal corresponding to event e_patrol_task (i.e. goal (false, e_patrol_task, false)). The bigraph pattern is

$$
\text { Goal.(SC.(False | id) | FC.(False | id) | Try.id) }
$$

where SC is the success condition, FC the failure condition, and Try the plan choice $\triangleright$. The presence of Try indicates that event e_patrol_task is within the given declarative goal and has been reduced to its set of relevant plans, from which an applicable plan is selected, according to the right-hand side of the reaction given in Fig. 13b. As long as Try is present (regardless of what is under it, i.e. Try.id), the declarative goal is being pursued. 


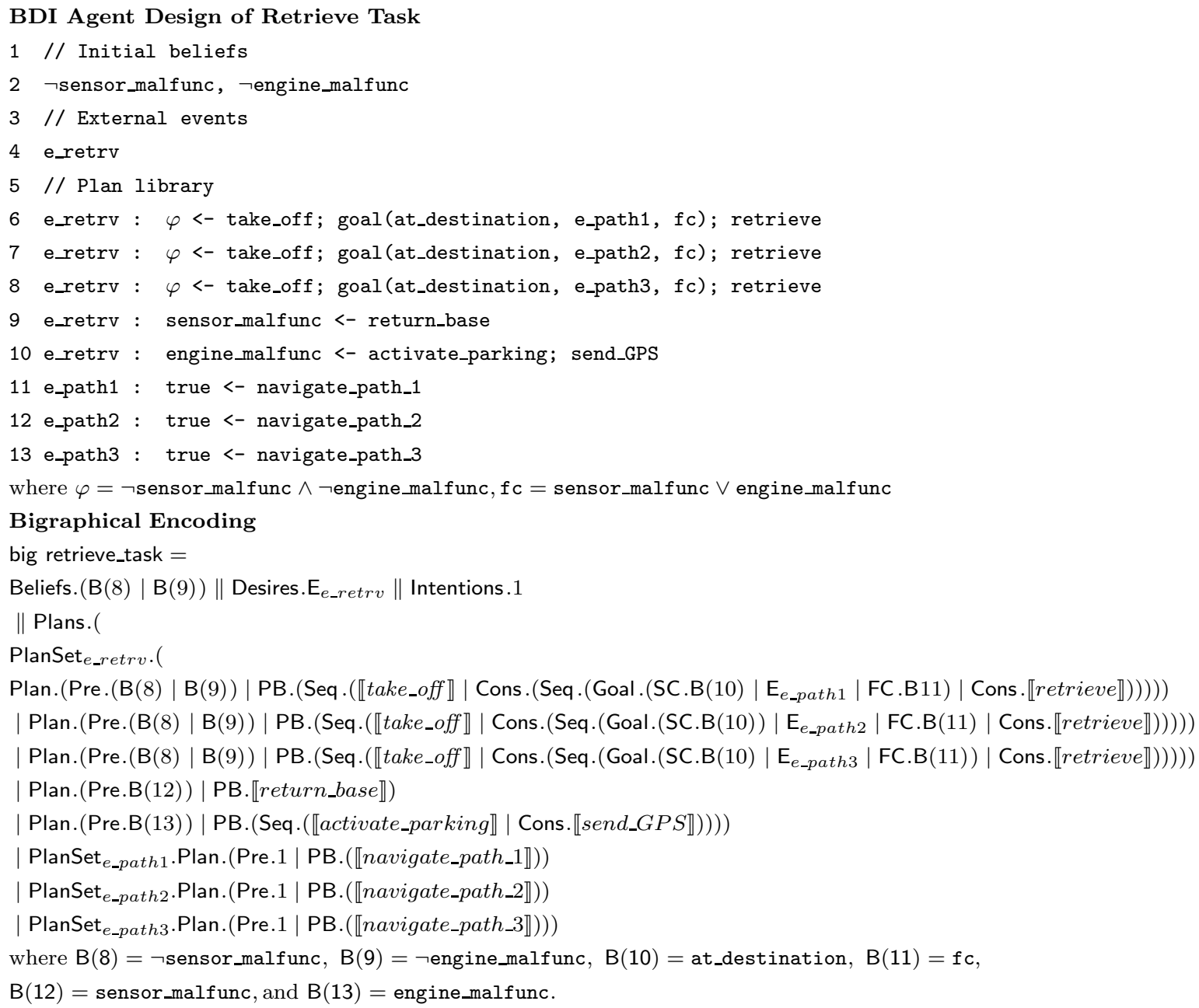

Figure 24: Retrieval Contingency: BDI Agent Design and Bigraphical Encoding. 


\subsection{Example properties}

Example 1 (Persistent patrol). A key property is that the goal corresponding to event e_patrol_task is persistent: $\mathbf{A}\left[\mathbf{G} \mathbf{F} \varphi_{1}\right]$, where

$$
\varphi_{1} \stackrel{\text { def }}{=} \text { Goal.(SC.(False | id) | FC.(False | id) | Try.id) }
$$

As expected the property holds.

Example 2 (Concurrent sensing) A useful property to investigate is whether it is possible to complete both sensing tasks regardless of their interleaving. Recall that in CAN semantics, whenever an intention is completed or fails, the agent will simply remove it from the intention base ( $A_{\text {update }}$ Fig. 16). Therefore, to make sure that an intention is successfully achieved, we have to ensure that a given intention is indeed removed only after being completed successfully. We denote the bigraph pattern for the successful completion of a given intention as $\varphi_{2} \stackrel{\text { def }}{=}$ Intent.1, the failure of completion of an intention $\varphi_{3} \stackrel{\text { def }}{=}$ Intent. ReduceF, and the removal of an intention from intention base $\varphi_{4} \stackrel{\text { def }}{=}$ Intentions.1. Bigraph pattern $\varphi_{4}$ specifies the removal of an intention from the intention base if and only if it is the only intention in the base. If there is more than one intention, it is impossible to reason about which intention is removed because there are no intention identifiers in CAN. We reflect on this lack in Section 9.6. Given the bigraph patterns above, the property is $\mathbf{A}\left[\mathbf{F}\left(\varphi_{2} \wedge \mathbf{X} \varphi_{4}\right)\right]$. This property is false, and we find that $\mathbf{E}\left[\mathbf{F}\left(\varphi_{3} \wedge \mathbf{X} \varphi_{4}\right)\right]$ holds (i.e. there exists a path for which eventually the intention is removed after being failed). This is because concurrency can introduce undesirable race conditions. For example, the action send_back in line 7 needs to be executed before the action save_shots is executed, to free required storage. This example highlights the benefits of a formal model for analysis at design time.

Example 3 (Contingency handling) Similar to example 2, a desirable property is that regardless of any malfunction, the intention for event e_retr is removed after successful completion. The property is $\mathbf{A}\left[\mathbf{F}\left(\varphi_{2} \wedge \mathbf{X} \varphi_{4}\right)\right]$ and it holds.

Before we give the results of the above property verification, we recall that our bigraph encoding introduces intermediate states that do not correspond to an agent step. Therefore, the operator $\mathbf{X}$ (next) has to be used carefully; some properties may require modification, because e.g. the next operator refers to the next internal state, not the next agent state. For example, there may be belief checks between agent steps. In the examples 2 and 3 above, no modifications were required.

\subsection{Results}

For automatic verification we exported the transition system to the PRISM model-checker 8 by assuming all transitions occur with equal probability. The

\footnotetext{
${ }^{8}$ Currently the only model-checker format supported by BigraphER.
} 
size of transition system generated by BigraphER and verification times are as follows:

\begin{tabular}{lllll} 
Example & States & Transitions & Build time (s) & Ver. time (s) \\
\hline Persistent patrol & 239 & 287 & 21.92 & 0.081 \\
Concurrent sensing & 731 & 879 & 61.16 & 0.01 \\
Contingency handling & 644 & 922 & 238.53 & 0.002
\end{tabular}

While contingency handling has fewer states/transitions than the concurrent sensing example, it takes more time to generate the transition system. We attribute this to the former containing more bigraph entities.

\section{Reflections on Can}

We reflect on the insights gained into the CAN language through the process of building the bigraph model. We stress that this should not be taken as criticism of CAN. On the contrary, we hope to show that the explicitness of the bigraph encoding is useful, e.g. to show areas of semantics with too much (resp. too little) information, and to aid in the continuous advancement of BDI family languages, in particular, from the point of verification and validation.

\subsection{Modularity in Semantics of CAN}

A unique characteristic of CAN is that it has a modular operational semantics that separates how to evolve an intention (i.e. the intention-level semantics) from how to evolve the whole agent (i.e.the agent-level semantics). This approach has its merits, for example, we can easily extend or modify one side of the semantics (e.g. the agent-level) without altering the other one. This was illustrated when adding the concurrency and declarative goals extensions (Section 6). The extensions only change intention-level steps, and as such, do not affect the overall faithfulness theorem as this is defined over agent-level steps.

The two-levels of semantics could be useful for verification. For example, we may consider only the agent-level transitions which would give snapshots of the agent state, without any information on how choices were made. In the bigraph model we do not make a distinction between agent-level and intentionlevel transitions, and both appear in the resulting output. The bridging of agent-level and intention-level transitions is performed by the introduction of the Reduce entity. For example, the reaction rule intention_step that encodes the agent-level derivation rule $A_{\text {steps }}$ introduces a Reduce entity to an intention, requesting it to be reduced according to the intention-level semantics. The use of instantaneous reaction rules, that do not show up in the resulting transition system, would allow only agent-level steps to be analysed without changes to the reaction rules themselves. 


\subsection{Inconsistency of Semantics in CAN Literature}

In the literature, there are subtle differences between definitions of the CAN semantics. In particular, between [6] and 21]. For example, consider the $\triangleright_{\perp}$ rule from the two works above:

$$
\begin{aligned}
& \frac{P_{1} \neq \text { nil } \quad\left\langle\mathcal{B}, P_{1}\right\rangle \nrightarrow}{\left\langle\mathcal{B}, P_{1} \triangleright P_{2}\right\rangle \rightarrow\left\langle\mathcal{B}, P_{2}\right\rangle} \quad \triangleright_{\perp} \text { in }[6] \\
& \frac{P_{1} \neq n i l \quad\left\langle\mathcal{B}, P_{1}\right\rangle \nrightarrow \quad\left\langle\mathcal{B}, P_{2}\right\rangle \rightarrow\left\langle\mathcal{B}^{\prime}, P_{2}^{\prime}\right\rangle}{\left\langle\mathcal{B}, P_{1} \triangleright P_{2}\right\rangle \rightarrow\left\langle\mathcal{B}^{\prime}, P_{2}^{\prime}\right\rangle} \quad \triangleright_{\perp} \text { in [21] }
\end{aligned}
$$

In [6] , the rule $\nabla_{\perp}$ is only dependent upon the irreducibility of the program $P_{1}$. However, in [21], not only is it dependent upon the irreducibility of $P_{1}$, but, within the same operation, the reducibility of $P_{2}$.

This change is significant as, in the first case, we wait to do failure recovery. This can allow the current belief base to be updated before selecting a new plan (in all cases $P_{2}$ has the form $e:(|\Delta|)$ ). In the second case there is no scope to wait for belief base changes.

It is not immediately clear which approach is better in practice. One benefit of a formal model is that we can begin to unpick these questions by substituting the current try_failure reaction for a modified version.

\subsection{Redundant Event Names}

The CAN language includes the form $e:(|\Delta|)$ representing a set of relevant plans which can be used to address the event $e$. This set is updated as plans are selected and executed. For example, when an applicable plan is selected (i.e. $\varphi: P \in \Delta$ and $\mathcal{B} \models \varphi$ ), it will be removed from the set of remaining plans (i.e. $e:(|\Delta \backslash\{\varphi: P\}|))$. However, after a set of relevant plans is selected from the plan library, the event name $e$ becomes redundant in the sense that it is never used by any CAN semantic rules. This is seen clearly in the bigraph model, where only reduce_event (in Fig. 12) utilises the event name link. Other rules always match the event name as open (connected to 0 or more other entities). This suggests that the form of plans within the plan library, and those within intentions should be different, e.g. $e$ : $(|\Delta|)$ and $(|\Delta|)$.

\subsection{No Difference between Intention Success and Failure}

As a high-level planning language, CAN remains agnostic to many important issues in practice. One such issue is the inability to tell if an intention completed successfully, or with a failure. The derivation rule $A_{\text {update }}$ in Fig. 7 simply removes a completed intention from the intention base, namely an intention nil or one that is failed and cannot make any further transition. Therefore, the completion of an intention is not equivalent to the achievement of an intention. To verify the achievement of an intention (which in practice is the most important property to check), we also have to ensure that its completion is not due to the failure. This is precisely how we verify the achievement of an intention in Section 8.2. Therefore, in our bigraph model, we have to encode the derivation rule $A_{\text {update }}$ into two cases, namely intention_done_F for failure case and intention_done_succ for success case. 


\subsection{Oracle for Failure}

Failure in CAN semantics is denoted by $\left\langle\mathcal{B}, P_{1}\right\rangle \nrightarrow$ as the premiss in the related derivation rule (e.g. $\nabla_{\perp}$ ). Therefore, to be able to apply the rule $\nabla_{\perp}$, the agent somehow can "look-ahead" to the result of the inner-reduction, i.e. there is some oracle that determines if the inner-reduction is possible. However, in practice (e.g. our bigraph encoding), no oracle exists, and the agent has to explicitly to try progress a step to see if it reduces. In others words, unlike the derivation rules which, to some extent, have the impression the failure occurs via one single rule $\left\langle\mathcal{B}, P_{1}\right\rangle \nrightarrow$, it actually involves a strict partial execution of other rules. It can be clearly seen in Fig. 17 where, before an action is deemed as un-executable, its pre-condition has to be actually checked to be false according to the belief base.

\subsection{Absence of Meta-level Reasoning}

While it is possible to reason about agents when only a single intention is involved - for example through checking a property that checks if an intention failed before it was removed (see Section 8.2) - these approaches do not apply when there are more than one concurrent intentions. The main issue here is that intentions lack identifiers. If we want to stay within the semantics in CAN, approaches to identifying specific intentions include (1) fixing the last program within any intention to be unique to allow checking when this specific program is removed, or (2) ensuring actions add unique beliefs however this requires knowing ahead of time the actions that will be executed in success/failure cases.

Ideally, intentions would have unique identifier to aid verification. Adding such an identifier is straightforward by replacing $P \in \Gamma$ with $\langle$ identifier, $P\rangle \in \Gamma$. As such, to track an intention is removed, we simply have a bigraph pattern Intent.(identifier | id) where identifier is the identifier of the intention and id the site that abstracts away specific details of the intention. We argue that by indexing the intentions and further labelling its status (e.g. active or suspended), the meta-level reasoning can be powerful for next level of agent verification, in particular, in the context of interacting with users.

Another area where keeping meta-information available is useful is to allow tracking events to the intentions that are handling those events. In the current semantics, when an event is processed (by $A_{\text {event }}$ ) it is removed completely from the desires structure and replaced by the set of relevant plans within an intention. As we execute the plans we lose track of which event $e$ generated that intention (i.e. the means-end relations).

\section{Related Work}

Reasoning about BDI agents through model checking has been well explored. A key work in this area 29] reports a translation of AgentSpeak programs to both the Promela modelling language and Java, and shows how to apply the Spin [30] model checker and Java PathFinder program model checker to verify the agents. Comparing the translation experience of these two, Java stood 
out as the most promising approach (as a general purpose language) compared to Promela (often used for the verification of communication protocols). Many have built upon this Java-based verification approach. In particular, recent work implements a BDI agent programming infrastructure as a set of Java classes the Agent Infrastructure Layer (AIL) 12]. The Gwendolen BDI language [31] provides the default semantics for the AIL, and is designed with verification in mind by including extra book-keeping and transition rules that purely assist verification.

The AIL has been further developed [32] to support the verification of heterogeneous multi-agent systems by allowing different agent programming languages to be used within the same AIL framework. Although the AIL supports heterogeneous agents, to date the BDI programming languages implemented in the AIL [33] is tightly bounded to Gwendolen and its extensions (e.g. 34]) along with another language named GOAL 35]. Crucially, what these approaches verify is the implementation of a given language. The faithfulness of the implementation to the language semantics is often omitted for convenience. Utilising Java PathFinder (and its enhanced version [32]) has the advantage of bypassing the need of a mathematical model by deriving the model directly from the program code. However it typically suffers from a significant performance bottleneck due to the symbolic execution of Java bytecode.

Agent properties for AIL are usually specified in linear temporal logic (LTL) [36], There is, however, an exception in [37] where the model generated by Java PathFinder is converted to the input language of PRISM 38] to, e.g. provide access to probabilistic property specification. Unfortunately the conversion to PRISM does not maintain direct link between the implemented program and the model being verified, e.g. it might be difficult to reflect back into the application when creating counter examples.

The two main BDI languages implemented in AIL are Gwendolen and GOAL. Unlike main-stream BDI programming languages, e.g. AgentSpeak, GOAL is a pure reactive system and does not select pre-defined plans from a library but instead selects individual actions. Like CAN, Gwendolen handles declarative goals, failure recovery and concurrency with some differences. In Gwendolen, declarative goals make statements about the beliefs the agent wishes to hold and remains a goal until the agent gains the appropriate beliefs. As such, the declarative information in Gwendolen is only carried for the initial goal of the intention, no declarative information is carried for any of its active sub-events. For example, if beliefs sought hold, the sub-event will still be executed to the end. Meanwhile, in CAN, the declarative information is carried for any stage of evolution of programs in the declarative goal. Once the success condition holds, the related program is halted immediately. Gwendolen does not allow goal failure conditions so is unable to decouple goal failure from plan failure. For failure recovery, Gwendolen is explicitly programmed with the appropriate plan revision rules (as meta-level rules) which specify a prefix of the current plan to be dropped and replaced by another. Finally, concurrency in Gwendolen is only allowed in the intention level, and, by default, is conducted in round-robin fashion to manage interleaving. 
Recent work continues to extend the verification framework above, improving the efficiency of verification and supporting richer property specifications. Program slicing has been used to increase verification efficiency by removing parts of the agent program that cannot affect the properties of interest [39]. Meanwhile, by simplifying the structure and execution of AgentSpeak (deviating from mainstream BDI agents), it can facilitate the verification of probabilistic and time bound properties through PRISM [40]. Finally, there is promising progress to verify the hybrid autonomous system in which the high-level is discrete logicbased framework (modelled by BDI agents) and the low-level is a continuous control system [41].

Bigraphs have been shown 22 to be suitable for encoding process algebras such as CCS [42], Mobile Process [43], $\pi$-calculus [15], and Actors [44]. Recently, there is also a growing trend to specify and verify agent-based systems via bigraphs, in particular, multi-agent systems. However, most of them still remain at the stage of proof of concept. For example, the work [45] proposes a methodology for modelling and simulating multi-agent systems via bigraphs. The core idea is that the containment relation of bigraphs mirrors the administrative relations of agents while reaction rules model agent reconfigurations, e.g. bigraph destruction translates into agent termination. One work that is perhaps closest to ours is [46], which also models BDI agents via bigraphs. However, it considers multi-agent systems, and treats the internal reasoning of each BDI agent as a black box. As a result, they provide no details regarding how the agents behave in an environment.

\section{Future Work}

The encoding of BDI agents in bigraphs is our first step laying out a foundation for more advanced reasoning. As future work, we have in mind two extensions: probabilistic reasoning, in particular, plan selection and intention trade-off, and dynamic environments.

In general, there may be several applicable plans which achieve a given event. The agent has to select one and it may be desirable to specify what is "most appropriate" at that time, which may depend upon different, and possibly domain-specific characteristics, e.g. cost and preference. Additionally, the agent may be pursuing a set of concurrent intentions, i.e. there is concurrency between the top-level external events. Similar to the plans, intention, it may be desirable to again specify "most appropriate" e.g. more urgent.

We will develop a more nuanced approach to handling plan selection and intention scheduling by assigning weights (to plans and events). These will be encoded by reaction rules with weights using probabilistic bigraphs [17] that export Discrete-time Markov chains (DTMCs).

Our current encoding of BDI agents is limited to a self-static environment, i.e. the environment changes only when the agent changes it. We plan to develop a self-dynamic environment and will extend the mechanism of failure recovery to allow re-selection of previously failed plans. This will not only increase the 
persistence of an agent, but also increase the likelihood of success by taking advantage of environmental changes.

\section{Conclusion}

Rational agents, such as Belief-Desire-Intention (BDI) agents, will play a key role in future autonomous systems and it is essential we can reason about their behaviours, and provide early, i.e. design-time, indications of potential problems, e.g. deadlocks causes by shared resources.

We have presented a framework, based on Milner's bigraphs, for modelling and verifying BDI agents specified in the CAN language. We believe this is the first executable semantics of CAN, allowing verification of abstract agent programs, rather than verification based on a specific implementation of CAN. The use of four perspectives in the bigraph model: Belief, Desire, Intention and Plan, helps is to separate concerns in the encoding and offers a clear visualisation of the resulting model.

The two key functions are the syntax encoding $\llbracket \cdot \rrbracket$ and $\mathbb{\|}$ that enables the behavioural encoding. The former has the added feature of introducing event indices for plans, which decreases errors and aids search. The latter is a bridge between agent-level and intention-level steps. Bigraph parallelism indicates how the belief base is the environment for reduction and conditional bigraphs allow us to prioritise reaction rules, which simplifies the encoding.

We have shown that the encoding of CAN agents in bigraphs is faithful by proving any CAN step is captured by a finite sequence of bigraph reaction rules, and we have shown shown the approach is practical through three example UAV applications. In each case, generating and verifying the model took no more than a few minutes.

This work has also highlighted many interesting features of the current semantics of CAN, such as the inability to distinguish between the success and failure of an intention and lack of meta-level reasoning, and it lays the foundation for future modelling work. We envisage an extended model (and hence extended semantics) that features probabilistic choice and dynamic environments - allowing quantitative model checking of agent programs.

\section{Acknowledgements}

This work is supported by the Engineering and Physical Sciences Research Council, under PETRAS SRF grant MAGIC (EP/S035362/1) and S4: Science of Sensor Systems Software. (EP/N007565/1)

\section{References}

[1] M. Bratman, Intention, Plans, and Practical reason. Harvard University Press, 1987. 
[2] A. S. Rao, "AgentSpeak (L): BDI agents speak out in a logical computable language," in Proceedings of European Workshop on Modelling Autonomous Agents in a Multi-Agent World. Springer, 1996, pp. 42-55.

[3] K. V. Hindriks, F. S. D. Boer, W. V. d. Hoek, and J.-J. C. Meyer, "Agent programming in 3APL," Autonomous Agents and Multi-Agent Systems, vol. 2, no. 4, pp. 357-401, 1999.

[4] M. Dastani, "2APL: a practical agent programming language," $A u$ tonomous agents and multi-agent systems, vol. 16, no. 3, pp. 214-248, 2008.

[5] R. Bordini, J. Hübner, and M. Wooldridge, Programming multi-agent systems in AgentSpeak using Jason. John Wiley \& Sons, 2007, vol. 8.

[6] S. Sardina, L. d. Silva, and L. Padgham, "Hierarchical planning in BDI agent programming languages: A formal approach," in Proceedings of the International Joint Conference on Autonomous Agents and Multiagent Systems, 2006, pp. 1001-1008.

[7] S. S. Benfield, J. Hendrickson, and D. Galanti, "Making a strong business case for multiagent technology," in Proceedings of the Fifth International Joint Conference on Autonomous Agents and Multiagent systems. ACM, 2006, pp. 10-15.

[8] L. Braubach, A. Pokahr, and W. Lamersdorf, "Negotiation-based patient scheduling in hospitals," in Advanced Intelligent Computational Technologies and Decision Support Systems, 2014, pp. 107-121.

[9] S. McArthur, E. Davidson, V. Catterson, A. Dimeas, N. Hatziargyriou, F. Ponci, and T. Funabashi, "Multi-agent systems for power engineering applications - part i: Concepts, approaches, and technical challenges," vol. 22 , no. 4 . IEEE, 2007, pp. 1743-1752.

[10] G. Brat, E. Denney, D. Giannakopoulou, J. Frank, and A. Jonsson, "Verification of autonomous systems for space applications," in Proceedings of IEEE Aerospace Conference, 2006.

[11] L. Lestingi, M. Askarpour, M. M. Bersani, and M. Rossi, "Formal verification of human-robot interaction in healthcare scenarios," in Proceedings of International Conference on Software Engineering and Formal Methods. Springer, 2020, pp. 303-324.

[12] L. A. Dennis, B. Farwer, R. H. Bordini, and M. Fisher, "A flexible framework for verifying agent programs," in Proceedings of the International Joint Conference on Autonomous Agents and Multiagent Systems, 2008, pp. 1303-1306.

[13] G. Brat, K. Havelund, S. Park, and W. Visser, "Model checking programs," in Proceedings of IEEE International Conference on Automated Software Engineering. IEEE, 2000, pp. 3-11. 
[14] R. Milner, "Bigraphs and their algebra," Electronic Notes in Theoretical Computer Science, vol. 209, pp. 5-19, 2008.

[15] M. Bundgaard and V. Sassone, "Typed polyadic pi-calculus in bigraphs," in Proceedings of ACM SIGPLAN International Conference on Principles and Practice of Declarative Programming, 2006, pp. 1-12.

[16] M. Sevegnani and M. Calder, "Bigraphs with sharing," Theoretical Computer Science, vol. 577, pp. 43-74, 2015.

[17] B. Archibald, M. Calder, and M. Sevegnani, "Probabilistic bigraphs," Submitted for publication, 2020.

[18] B. Archibald, C. Muffy, and M. Sevegnani, "Conditional bigraphs," in International Conference on Graph Transformation. Springer, 2020, pp. $3-19$.

[19] M. Sevegnani and M. Calder, "Bigrapher: rewriting and analysis engine for bigraphs," in Proceedings of International Conference on Computer Aided Verification. Springer, 2016, pp. 494-501.

[20] S. Benford, M. Calder, T. Rodden, and M. Sevegnani, "On lions, impala, and bigraphs: Modelling interactions in physical/virtual spaces," $A C M$ Transactions on Computer-Human Interaction (TOCHI), vol. 23, no. 2, pp. 1-56, 2016.

[21] S. Sardina and L. Padgham, "A BDI agent programming language with failure handling, declarative goals, and planning," Autonomous Agents and Multi-Agent Systems, pp. 18-70, 2011.

[22] R. Milner, The space and motion of communicating agents. Cambridge University Press, 2009.

[23] M. Calder and M. Sevegnani, "Modelling IEEE 802.11 CSMA/CA RTS/CTS with stochastic bigraphs with sharing," Formal Aspects of Computing, vol. 26, no. 3, pp. 537-561, 2014.

[24] G. D. Plotkin, "A structural approach to operational semantics," in Lecture Notes, Aarhus University Denmark, 1981.

[25] B. Logan, J. Thangarajah, and N. Yorke-Smith, "Progressing intention progression: A call for a goal-plan tree contest," in Proceedings of International Conference on Autonomous Agents and Multiagent Systems, 2017, pp. $768-772$.

[26] M. Xu, K. McAreavey, K. Bauters, and W. Liu, "Intention interleaving via classical replanning," in Proceedings of International Conference on Tools with Artificial Intelligence, 2019, pp. 85-92. 
[27] B. Archibald, M. Calder, M. Sevegnani, and M. Xu, "Modelling and verifying bdi agents with bigraphs - models," Jan. 2020. [Online]. Available: https://doi.org/10.5281/zenodo.4472541

[28] E. M. Clarke and E. A. Emerson, "Design and synthesis of synchronization skeletons using branching time temporal logic," in Proceedings of Workshop on Logic of Programs. Springer, 1981, pp. 52-71.

[29] R. H. Bordini, M. Fisher, W. Visser, and M. Wooldridge, "Verifying multiagent programs by model checking," Autonomous Agents and Multiagent Systems, vol. 12, no. 2, pp. 239-256, 2006.

[30] G. J. Holzmann, "The model checker spin," IEEE Transactions on software engineering, vol. 23, no. 5, pp. 279-295, 1997.

[31] L. A. Dennis, "Gwendolen semantics: 2017," Technical Report ULCS-17001, University of Liverpool, 2017.

[32] L. A. Dennis, M. Fisher, M. P. Webster, and R. H. Bordini, "Model checking agent programming languages," Automated software engineering, vol. 19, no. 1 , pp. 5-63, 2012.

[33] L. A. Dennis, "The mcapl framework including the agent infrastructure layer and agent java pathfinder," The Journal of Open Source Software, 2018.

[34] L. Dennis, M. Fisher, M. Slavkovik, and M. Webster, "Formal verification of ethical choices in autonomous systems," Robotics and Autonomous Systems, vol. 77, pp. 1-14, 2016.

[35] K. V. Hindriks, F. S. De Boer, W. Van Der Hoek, and J.-J. C. Meyer, "Agent programming with declarative goals," in Proceedings of International Workshop on Agent Theories, Architectures, and Languages. Springer, 2000, pp. 228-243.

[36] E. A. Emerson, "Temporal and modal logic," in Formal Models and Semantics. Elsevier, 1990, pp. 995-1072.

[37] L. A. Dennis, M. Fisher, and M. Webster, "Two-stage agent program verification," Journal of Logic and Computation, vol. 28, no. 3, pp. 499-523, 2018.

[38] M. Kwiatkowska, G. Norman, and D. Parker, "Prism 4.0: Verification of probabilistic real-time systems," in Proceedings of International conference on computer aided verification. Springer, 2011, pp. 585-591.

[39] M. Winikoff, L. Dennis, and M. Fisher, "Slicing agent programs for more efficient verification," in International Workshop on Engineering Multi-Agent Systems. Springer, 2018, pp. 139-157. 
[40] P. Izzo, H. Qu, and S. M. Veres, "A stochastically verifiable autonomous control architecture with reasoning," in Proceedings of IEEE Conference on Decision and Control. IEEE, 2016, pp. 4985-4991.

[41] L. A. Dennis, M. Fisher, N. K. Lincoln, A. Lisitsa, and S. M. Veres, "Practical verification of decision-making in agent-based autonomous systems," Automated Software Engineering, vol. 23, no. 3, pp. 305-359, 2016.

[42] R. Milner, "Pure bigraphs: structure and dynamics," Information and computation, vol. 204, no. 1, pp. 60-122, 2006.

[43] O. H. Jensen, "Mobile processes in bigraphs," Ph.D. dissertation, University of Aalborg, 2006.

[44] M. Sevegnani and E. Pereira, "Towards a bigraphical encoding of actors," in Proceedings of International Workshop on Meta Models for Process Languages, 2014.

[45] A. Mansutti, M. Miculan, and M. Peressotti, "Multi-agent systems design and prototyping with bigraphical reactive systems," in Ifip international conference on distributed applications and interoperable systems. Springer, 2014, pp. 201-208.

[46] A. T. E. Dib and Z. Sahnoun, "Model checking of multi-agent system architectures using bigmc," in Proceedings of Federated Conference on Computer Science and Information Systems, 2015, pp. 1717-1722. 